\title{
Hyaluronidase and Hyaluronan Oligosaccharides Promote Neurological Recovery after Intraventricular Hemorrhage
}

\author{
Govindaiah Vinukonda, ${ }^{1}$ Preeti Dohare, ${ }^{1}$ OArslan Arshad, ${ }^{1}$ Muhammad T. Zia, ${ }^{1}$ Sanjeet Panda, ${ }^{1}$ Ritesh Korumilli, ${ }^{1}$ \\ Robert Kayton, ${ }^{3}$ Vincent C. Hascall, ${ }^{4}$ Mark E. Lauer, ${ }^{4{ }^{\dagger}}$ and Praveen Ballabh ${ }^{1,2 *}$ \\ Departments of ${ }^{1}$ Pediatrics and ${ }^{2}$ Cell Biology and Anatomy, Regional Neonatal Center, Maria Fareri Children's Hospital at Westchester Medical Center, New \\ York Medical College, Valhalla, New York 10595, ${ }^{3}$ Department of Physiology and Pharmacology, Oregon Health \& Science University, Portland, Oregon \\ 97239, and ${ }^{4}$ Department of Biomedical Engineering, Cleveland Clinic, Cleveland, Ohio 44195
}

Intraventricular hemorrhage (IVH) in premature infants results in inflammation, arrested oligodendrocyte progenitor cell (OPC) maturation, and reduced myelination of the white matter. Hyaluronan (HA) inhibits OPC maturation and complexes with the heavy chain (HC) of glycoprotein inter- $\alpha$-inhibitor to form pathological HA (HC-HA complex), which exacerbates inflammation. Therefore, we hypothesized that IVH would result in accumulation of $\mathrm{HA}$, and that either degradation of $\mathrm{HA}$ by hyaluronidase treatment or elimination of HCs from pathological HA by HA oligosaccharide administration would restore OPC maturation, myelination, and neurological function in survivors with IVH. To test these hypotheses, we used the preterm rabbit model of glycerol-induced IVH and analyzed autopsy samples from premature infants. We found that total $\mathrm{HA}$ levels were comparable in both preterm rabbit pups and human infants with and without IVH, but HA receptors-CD44, TLR2, TLR4 - were elevated in the forebrain of both humans and rabbits with IVH. Hyaluronidase treatment of rabbits with IVH reduced CD44 and TLR4 expression, proinflammatory cytokine levels, and microglia infiltration. It also promoted OPC maturation, myelination, and neurological recovery. HC-HA and tumor necrosis factor-stimulated gene-6 were elevated in newborns with IVH; and depletion of $\mathrm{HC}-\mathrm{HA}$ levels by $\mathrm{HA}$ oligosaccharide treatment reduced inflammation and enhanced myelination and neurological recovery in rabbits with IVH. Hence, hyaluronidase or HA oligosaccharide treatment represses inflammation, promotes OPC maturation, and restores myelination and neurological function in rabbits with IVH. These therapeutic strategies might improve the neurological outcome of premature infants with IVH.

Key words: hyaluronan; hyaluronan oligosaccharides; hyaluronidase; microglia; myelination; oligodendrocyte

Significance Statement

Approximately 12,000 premature infants develop IVH every year in the United States, and a large number of survivors with IVH develop cerebral palsy and cognitive deficits. The onset of IVH induces inflammation of the periventricular white matter, which results in arrested maturation of OPCs and myelination failure. HA is a major component of the extracellular matrix of the brain, which regulates inflammation through CD44 and TLR2/4 receptors. Here, we show two mechanism-based strategies that effectively enhanced myelination and neurological recovery in preterm rabbit model of IVH. First, degrading HA by hyaluronidase treatment reduced CD44 and TLR4 expression, proinflammatory cytokines, and microglial infiltration, as well as promoted oligodendrocyte maturation and myelination. Second, intraventricular injection of HA oligosaccharide reduced inflammation and enhanced myelination, conceivably by depleting $\mathrm{HC}-\mathrm{HA}$ levels.

\section{Introduction}

Intraventricular hemorrhage (IVH) remains a major cause of neonatal white matter (WM) injury in premature infants. No

Received Aug. 31, 2015; revised Nov. 5, 2015; accepted Dec. 3, 2015.

Author contributions: G.V. and P.B. designed research; G.V., P.D., A.A., M.T.Z., S.P., R. Korumilli, M.E.L., and P.B. performed research; G.V., R. Kayton, M.E.L., and P.B. analyzed data; V.C.H., M.E.L., and P.B. wrote the paper.

This work was supported by NIH-NINDS Grants R01 NS071263 (P.B.) and R01NS083947 (P.B.), National Heart, Lung, and Blood Institute Grant HL113325 (M.E.L., V.C.H.), and a scientist development grant from the American Heart Association (G.V.). We thank Joanne Abrahams for the assistance with images. We thank Ron Midura from the Cleveland Clinic for assistance with the size exclusion chromatography and for his helpful consultations. We thank therapeutic strategy exists to prevent cerebral palsy and cognitive deficits in the survivors of IVH. In human premature infants, white matter lesions display an abundance of hyaluronan (HA), overexpression of HA receptor CD44, arrested maturation of oli-

Valbona Cali from the Cleveland Clinic for assistance with the fluorophore-assisted carbohydrate electrophoresis analysis, hyaluronan ELISA-like assay, and size exclusion chromatography by agarose and gel filtration, and for the measurement of hyaluronan heavy chains from brain tissues.

*P.B. and M.E.L. contributed equally to this work.

tDeceased, 0ct. 12, 2015.

The authors declare no competing financial interests. 
godendrocyte precursor cells (OPCs), and reduced myelination (Buser et al., 2012). HA inhibits OPC maturation and myelination in animal models of demyelinating brain lesions and in cell culture experiments (Preston et al., 2013). Therefore, we asked whether the development of IVH in preterm infants would result in accumulation of a specific modification of $\mathrm{HA}$ in the periventricular white matter and whether breakdown of HA by hyaluronidase treatment or reversing specific modification of HA would restore myelination and neurological function in infants with IVH.

HA is a negatively charged glycosaminoglycan polymer, which accumulates in the white matter lesions of premature infants, adult stroke, multiple sclerosis, traumatic spinal cord injury, and vanishing white matter disease (Struve et al., 2005; Al'Qteishat et al., 2006; Cargill et al., 2012). HA fragments of low molecular weight (LMW) and high molecular weight (HMW) exhibit a wide range of biological functions. HMW species can be immunosuppressive, antiangiogenic, and protective, whereas LMW HA fragments can be proinflammatory and angiogenic (Stern et al., 2006). Nonetheless, HMW HA $\left(\sim 0.9 \times 10^{6} \mathrm{Da}\right)$ inhibits remyelination in a mouse model of lysolecithin-induced white matter demyelination, but not LWM HA $\left(\sim 3 \times 10^{5} \mathrm{Da}\right)$ (Back et al., 2005). HA interacts with CD44 and TLR2/4 receptors, which are predominantly expressed on astrocytes and OPCs (Sloane et al., 2010; Buser et al., 2012). TLR2/4 receptors are activated in cerebral ischemia, trauma, hemorrhage, and infection (Konat et al., 2006; Fischer and Ehlers, 2008; Rivest, 2009), and inhibition of TLR2/4 offers neuroprotection in several adult rodent models of brain injury (Lehnardt et al., 2007; Tang et al., 2007). CD44 levels are chronically elevated in demyelinating lesions of multiple sclerosis, and antagonizing CD44 promotes remyelination and neurological recovery (Tuohy et al., 2004; Guan et al., 2011). Hence, specific fragments of HA can induce white matter injury, which can potentially be reversed by degrading HA or by blocking its binding receptors.

IVH in premature newborns induces inflammation, and $\mathrm{COX} 2$ or TNF $\alpha$ inhibition alleviates inflammation and promotes myelination along with neurological recovery in rabbits with IVH (Vinukonda et al., 2010). Proinflammatory cytokines have been shown to induce expression of tumor necrosis factor-stimulated gene-6 (TSG-6) in inflammatory disorders such as rheumatoid arthritis and sepsis (Milner and Day, 2003). TSG-6 catalyzes the covalent transfer of heavy chains (HCs) from inter- $\alpha$ trypsin inhibitor (I $\alpha \mathrm{I})$ proteoglycan to HA to form HC-HA complexes (Milner and Day, 2003). HC-HA complexes exhibit proinflammatory activity by promoting adhesion of leukocytes to HA matrices and by extravasation of leukocytes into the interstitial spaces (Zhuo et al., 2006). HA oligosaccharides of 8-21 monosaccharides $(\sim 1.5 \mathrm{kDa})$ have been used to remove the HCs from HC-HA complexes, thereby reducing the concentration of pathological HC-HA (Lauer et al., 2013b). HA oligosaccharides also impede HA-CD44 interaction by inducing CD44 receptor cleavage and by competitive binding to CD44 (Sugahara et al., 2003; Teriete et al., 2004). Indeed, HA oligosaccharide treatment offers neuroprotection in a mouse model of experimental allergic encephalitis (Winkler et al., 2013).

Although HA and myelination have been studied in adult animal models of demyelination, the dynamics of HA synthesis

Correspondence should be addressed to Dr. Praveen Ballabh, Regional Neonatal Center, Maria Fareri Children's Hospital at Westchester Medical Center, Valhalla, NY 10595. E-mail: pballabh@msn.com.

DOI:10.1523/JNEUROSCI.3297-15.2016

Copyright $\odot 2016$ the authors $\quad 0270-6474 / 16 / 360873-18 \$ 15.00 / 0$ and degradation and the effect of HA on myelination have not been evaluated in a developmental model of brain injury in premature newborns. Therefore, we hypothesized that IVH would result in the accumulation of specific fragments of HA and that degradation of HA by hyaluronidase treatment might reduce inflammation and restore OPC maturation and myelination in preterm survivors of IVH. We also postulated that TSG-6 activity is increased in IVH, resulting in increased production of pathological HC-HA, and that eliminating HCs from HC-HA complexes by treatment with HA oligosaccharides might reduce cerebral inflammation and enhance myelination as well as clinical recovery.

\section{Materials and Methods}

Animals. This study was approved by the Institutional Animal Care and Use Committee of the New York Medical College (Valhalla, NY). We used our well-established preterm rabbit model of glycerol-induced IVH for the study (Chua et al., 2009; Vinukonda et al., 2010; Dummula et al., 2011). Briefly, we purchased timed-pregnant New Zealand white rabbits from Charles River Laboratories. We performed Cesarean sections to deliver the premature pups at embryonic day 29 (E29; full term, 32 d). Newborn pups were reared in an infant incubator at a temperature of $35^{\circ} \mathrm{C}$. We used puppy formula (Zoologic) to gavage feed the pups in a volume of $\sim 2 \mathrm{ml}$ every $12 \mathrm{~h}(100 \mathrm{ml} / \mathrm{kg} / \mathrm{d})$ for the first $2 \mathrm{~d}$, and feeds were advanced to $125,150,200,250$ and $280 \mathrm{ml} / \mathrm{kg}$ at postnatal day 3 (P3), P5, $\mathrm{P} 7, \mathrm{P} 10$, and P14, respectively. We treated rabbit pups of either sex with $50 \%$ intraperitoneal glycerol $(6.5 \mathrm{gm} / \mathrm{kg}$ ) at $3 \mathrm{~h}$ of age to induce IVH (Fig. 1). Severity of IVH was determined by measuring ventricle volume by head ultrasound at $24 \mathrm{~h}$ age using an Acuson Sequoia C256 (Siemens) ultrasound machine. Pups were classified as moderate $\left(30-150 \mathrm{~mm}^{3}\right)$ and severe (151-250 $\mathrm{mm}^{3}$ ) IVH, based on ventricular volume. Ventricular volume of $<30 \mathrm{~mm}^{3}$ indicated microscopic or no IVH

Hyaluronidase and HA oligosaccharide (10 monosaccharide unit) treatment. The rabbit pups with IVH were sequentially treated with either 20 $\mu \mathrm{l}$ of hyaluronidase from Streptomyces hyalurolyticus (hyaluronidase, 1 $\mathrm{U} / \mu \mathrm{l}$; Sigma) or vehicle (saline) intracerebroventricularly at days 2,4 , and 6. Briefly, the pups were mounted on a rabbit pup restrainer after anesthetizing them with ketamine and xylazine. A $50 \mu$ l Hamilton syringe with a 27 gauge needle was mounted on a micromanipulator to inject the hyaluronidase into the lateral ventricle. We used the following coordinates from bregma: $1 \mathrm{~mm}$ anterior, $4 \mathrm{~mm}$ lateral, and $3 \mathrm{~mm}$ deep. The dose of hyaluronidase was calculated based on its previous use in rats to treat spinal cord and cerebral injury and in our initial experiments, showing major reduction in HA after hyaluronidase treatment. The severity of IVH, measured by ultrasound, was similar between the comparison groups. In another set of experiments, HA oligosaccharide (10 monosaccharide unit; Hyalose) in a dose of $10 \mu \mathrm{g}$ or vehicle (saline) was injected into each of the two lateral ventricles, in the same fashion as the hyaluronidase.

Human subjects. The Research Administration of New York Medical College (Valhalla, NY) approved the use of autopsy brain samples from premature infants for this study. The postmortem materials included forebrain tissue harvested from premature infants [23-27 gestational weeks (gw)] with and without IVH of $<5 \mathrm{~d}$ of postnatal age and $<18 \mathrm{~h}$ postmortem (Table 1 ). We excluded premature infants with hypoxic-ischemic encephalopathy, meningitis, culture proven sepsis, major brain malformation, and chromosomal defects. In this study, we described intermediate zone embryonic white matter synonymously with white matter, and cerebral cortex for the cortical plate (Bystron et al., 2008).

Immunohistochemistry. Approximately 3-mm-thick coronal slices at the head of caudate nucleus from human brains and 2-mm-thick slices taken at the level of the midseptal nucleus from rabbit forebrains were processed and immunolabeled as described previously (Dummula et al., 2011). The primary antibodies used in experiments included rabbit hyaluronan synthase (HAS) 1 (HAS1; AIVA System Biology), mouse HAS2 (Santa Cruz Biotechnology), rabbit HAS3 (AIVA System Biology), mouse 1 caspase 3 (Thermo Fisher Scientific), goat Ki67 (Cell Marque), 

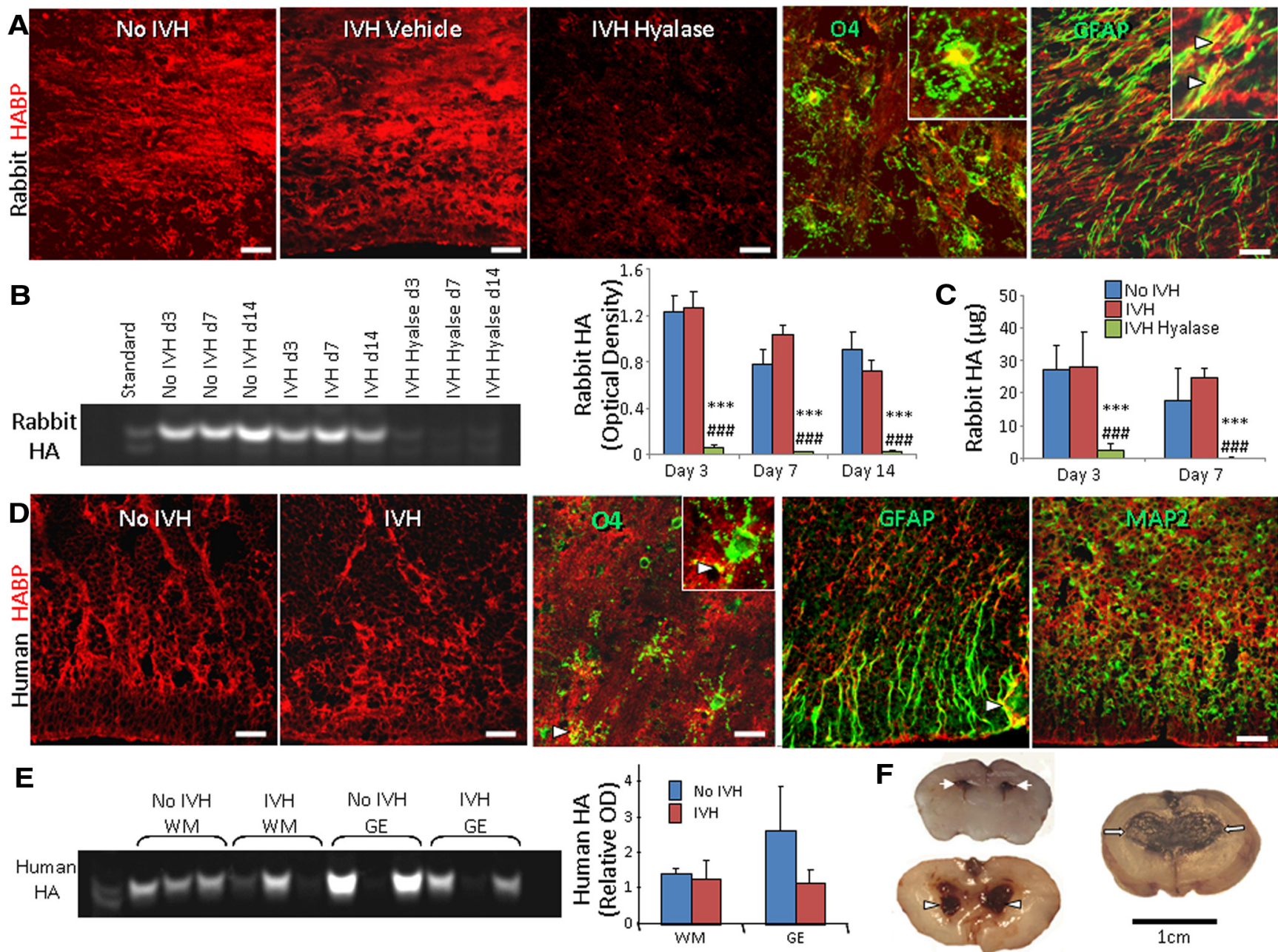

Figure 1. IVH does not affect total HA levels in preterm rabbits and humans. A, Cryosections from the forebrain of rabbit pups were labeled with HABP. Note comparable HA between sections from pups with and without IVH in periventricular white matter and lack of HA reactivity in hyaluronidase-treated pups. Few GFAP ${ }^{+}$astrocytes coexpressed $\mathrm{HA}$ (arrowheads). However, $04^{+} \mathrm{OPC}$ did not colocalize with HA signals. $\boldsymbol{B}$, Representative fluorophore-assisted carbohydrate electrophoresis on rabbit forebrain homogenates showing similar levels of HA in pups with and without IVH at days 3,7 , and 14. However, levels of HA were low in hyaluronidase-treated animals. Data are mean \pm SEM ( $n=6-11$ each group). HA levels were significantly reduced in hyaluronidase-treated pups. ${ }^{* * *} p<0.001$ (no IVH controls vs hyalase-treated pups with IVH); ${ }^{* \# \#} p<0.001$ (IVH pups vs hyalase-treated pups with IVH). C, HA levels were measured by ELISA and were found comparable between pups with and without IVH at both days 3 and 7 . However, HA levels were markedly reduced in hyaluronidase-treated pups. ${ }^{* * *} p<0.01$ (no IVH vs hyalase); ${ }^{\# \# \# ~} p<0.01$ (no IVH vs hyalase); mean \pm SEM. D, Representative immunofluorescence of cryosections from $23 \mathrm{gw}$ infants labeled with HABP show similar reactivity to HABP in infants with and without IVH. Immunoreactivities of few GFAP ${ }^{+}$astrocytes overlapped with $\mathrm{HA}$ (arrowhead). However, $04^{+} \mathrm{OPCs}$ and $\mathrm{MAP2}{ }^{+}$neurons did not colocalize with HA signals. E, Representative fluorophore-assisted carbohydrate electrophoresis on forebrain homogenates from preterm infants. Data are mean \pm SEM ( $n=4$ each group). HA levels were similar in infants with and without IVH. $\boldsymbol{F}$, Coronal brain slice through frontal lobe of E29 rabbit kit show normal slit-like ventricle (arrows, top), moderate hemorrhage in the ventricle (arrowheads, middle), and severe hemorrhage resulting in fusion of the two ventricles (arrows, bottom). Scale bars: $A, D, 20 \mu \mathrm{m} ; \boldsymbol{F}, 1 \mathrm{~cm}$.

goat Olig2 (R \& D Systems), mouse GFAP (Sigma), rat myelin basic protein (MBP; Abcam), mouse adenomatous polyposis coli (EMD Chemicals), goat PDGFR $\alpha$ (R \& D Systems), mouse myelin-associated glycoprotein (MAG; Abcam), mouse CD44 (Novus Biologicals), mouse TLR2 (Abcam), mouse TLR4 (Abcam), biotinylated O4 monoclonal antibody (from Dr. Rashmi Bansal, Farmington University of Connecticut), goat Ibal (Abcam), and mouse CD11B (AbD Serotec), goat TSG-6 antibody (R \& D Systems), and rabbit inter- $\alpha$-inhibitor (Dako).

Western blot analyses. Western blot analyses were performed as described previously (Ballabh et al., 2007). Briefly, homogenates made from brain slice taken at the level of the midseptal nucleus were quantified for protein using a BCA protein assay kit (Thermo Fisher Scientific). Equal amounts of protein $(10-20 \mu \mathrm{g})$ were loaded onto $4-15 \%$ or $4-20 \%$ gradient precast gels (Bio-Rad), depending on the molecular weight of the target protein. Separated proteins were transferred onto polyvinylidene difluoride membranes by electrotransfer. Membranes were incubated overnight with primary antibodies and target proteins were detected with an ECL system by using secondary antibodies conju- gated with horseradish peroxidase (Jackson Immunoresearch). The blots from each experiment were run two to three times and were densitometrically analyzed using ImageJ. Optical density values were normalized to $\beta$ actin.

Estimation of hyaluronan by fluorophore-assisted carbohydrate electrophoresis. HA contents in rabbit brain slice were measured as described previously (Lauer et al., 2009). Briefly, forebrain slices taken at the level of the midseptal nucleus were digested with proteinase $\mathrm{K}$, and HA was recovered by ethanol precipitation. The HA was digested to disaccharides at $37^{\circ} \mathrm{C}$ overnight using hyaluronidase SD (Seikagaku America) and then labeled with 2-aminoacridone (Life Technologies). The labeled HA disaccharides were separated by gel electrophoresis using a Bio-Rad miniPROTEAN Tetra system. After electrophoresis, the gels were imaged in their plates on a UV transilluminator (myEC imager; Thermo Fisher Scientific) at $365 \mathrm{~nm}$ using a CCD camera. The HA disaccharide bands were quantified using ImageJ.

Quantification of HA by ELISA-like assay and analysis of hyaluronan molecular weight distribution by agarose gel electrophoresis. HA, extracted 
Table 1. Characteristics of human infants with and without IVH

\begin{tabular}{lllll}
\hline $\begin{array}{l}\text { Postconceptional } \\
\text { age (weeks) }\end{array}$ & Sex & $\begin{array}{l}\text { Birth weight } \\
(\mathrm{kg})\end{array}$ & IVH/no IVH & Cause of death \\
\hline $26^{a}$ & Male & 0.810 & IVH grade 3 & Clinical sepsis \\
$23^{a}$ & Male & 0.57 & IVH grade 2 & Clinical sepsis \\
$23^{a}$ & Female & 0.58 & IVH grade 3 & Respiratory failure \\
$24^{a}$ & Male & 0.64 & IVH grade 4 & Pulmonary hemorrhage \\
24 & Male & 0.6 & IVH grade 4 & Clinical sepsis \\
23 & Female & 0.52 & IVH grade 3 & Respiratory failure \\
23 & Female & 0.53 & no IVH & Respiratory failure \\
25 & Female & 0.71 & no IVH & Metabolic acidosis, \\
& & & & respiratory failure \\
$23^{a}$ & Male & 0.45 & no IVH & RDS, respiratory failure \\
$24^{a}$ & Male & 0.61 & no IVH & Clinical sepsis \\
$25^{a}$ & Male & 0.73 & no IVH & Metabolic acidosis, \\
& & & & respiratory failure \\
$24^{a}$ & Female & 0.56 & no IVH & Respiratory failure \\
\hline
\end{tabular}

${ }^{a}$ Indicates autopsy samples used for experiments shown in Figure 6. RDS, Respiratory distress syndrome.

from rabbit brains by proteinase $\mathrm{K}$ digestion, was quantified by ELISAlike assay, as described previously (Haserodt et al., 2011). Agarose gel electrophoresis was performed on $2 \mathrm{~mm}$ coronal slices taken from the forebrain at the level of the midseptal nucleus, based on a previous description (Lauer et al., 2009).

Size exclusion chromatography. Briefly, HA was extracted from rabbit brains by proteinase $\mathrm{K}$ digestion and purified by ethanol precipitation. Nucleic acids were subsequently digested with Benzonase (EMD Millipore) and purified by ethanol precipitation. Chromatography columns were packed with Sephacryl S-1000 and equilibrated in $0.5 \mathrm{M}$ ammonium acetate. Calibration was accomplished by running 2500 , $1000,500,250,100$, and $10 \mathrm{kDa}$ Select-HA standards (Hyalose) on the columns. The mobile phase was pumped at $0.5 \mathrm{ml} / \mathrm{min}$, collecting 1 $\mathrm{ml}$ fractions in borosilicate tubes in an automated fraction collector. An aliquot of each fraction was lyophilized to dryness, resuspended in $0.1 \mathrm{~m}$ ammonium acetate, $\mathrm{pH} 7.0$, and digested with Streptomyces hyaluronidase overnight at $37^{\circ} \mathrm{C}$. The samples were then lyophilized to dryness, labeled with 2-aminoacridone, and incubated overnight at $37^{\circ} \mathrm{C}$. The samples were analyzed by electrophoresis and the bands quantified using Image J software, as described previously (Lauer et al., 2013a).

Stereological assessment of myelin and astrocytes in the WM and electron microscopy. These evaluations were performed as in our prior publication (Dummula et al., 2011).

$R T-q P C R$. Gene expression was performed by real time PCR on coronal slices taken from forebrain at the level of the midseptal nucleus, as described previously (Ballabh et al., 2007). The nucleotide sequence of primers used for RT-qPCR using SYBR green and TaqMan chemistry are described in Table 2.

Statistics and analysis. Data are expressed as means \pm SEM. For studies in rabbits, the expression of MBP, MAG, CNPase, and GFAP and cell counts were compared between three groups [no IVH, vehicle-treated, and hyaluronidase- or HA oligosaccharide of 10 monosaccharide length (HA10)treated pups] using one-way ANOVA. HA, gene expressions of bHLH transcription factors, TNF $\alpha$, CD44, TLR2/4, interferon $\gamma$, TSG-6, and protein levels of MBP were compared between three groups at days 3 and 7 using two-way ANOVA with repeated measures. In addition, differences in HAS1-HAS3 expression between pups with and without IVH were compared at days 3 and 7 by two-way ANOVA with repeated measures. For studies on human tissues, we compared HAS1-HAS3, HA, CD34, TLR2/4, HC-HA, and TSG activity between infants with and without IVH in three brain regions (cortex, white matter, and germinal matrix), using two-way ANOVA with repeated measures. The repeated factor was applied to the brain regions. All post hoc comparisons were done by Bonferroni's comparison test at the 0.05 significance level.
Table 2. Nucleotide sequence of primers

\begin{tabular}{|c|c|c|c|}
\hline & Accession \# & Sense & Antisense \\
\hline HAS1 & NM_001523.2 & TGTGTATCCTGCATCAGCGGT & $\begin{array}{l}\text { CTGGAGGTGTACTTGGTAGCA } \\
\text { TAACC }\end{array}$ \\
\hline HAS2 & NM_001082010 & TTCAGTGAAGTCATGGGCAGGGAA & $\begin{array}{l}\text { GTTCGACAAGACCAGTTGGGT } \\
\text { TAC }\end{array}$ \\
\hline HAS3 & NM_001082709.1 & GGTACCATCAGAAGTTCCTAGGCAGC & GAGGAGAATGTTCCAGATGCG \\
\hline TLR2 & NM_001082781.1 & AGGTGCCTCCTTGTTACCTATGCT & $\begin{array}{l}\text { AGATGAAGTTGTTCCCTCCGGC } \\
\text { TT }\end{array}$ \\
\hline TSG-6 & NM_001082311.1 & ACTCAAGTATGGTCAGCGTATTC & TCTCCACAGTACCTTCCTACAA \\
\hline GFAP & NG_008401 & ACTCAATGCTGGCTTCAAGGAGAC & $\begin{array}{l}\text { ATGTAGCTGGCAAAGCGGTCA } \\
\text { TTG }\end{array}$ \\
\hline Id2 & XM_002723742.1 & CCATGAGCCTGCTCTACAA & GTGCTGCAGGATTTCCATTT \\
\hline Id4 & NM_001546 & GGCATAATGGCAAATCCTTCAAG & TCACAAGAGATGGGACAGTAGC \\
\hline Olig1 & XM_002716810.1 & AGGTCATCCTGCCCTACTC & CCAGCAGCAGGATGTAGTT \\
\hline Olig2 & XM_002716698.1 & TTCAAGTCCTCCTCGTCCA & GGCTCGGTCATCTGTTTCTT \\
\hline Sox10 & XM_002723532 & AAGCCTTTCTGTCTGGCTCACT & $\begin{array}{l}\text { TCAGGTCCTGGATAGAGGGT } \\
\text { CATT }\end{array}$ \\
\hline GAPDH & NM_001082253.1 & GCGTGAACCACGAGAAGTAT & ССТCCACAATGCCGAAGT \\
\hline
\end{tabular}

The gene expression for hyaluronidase 1 (assay ID, Hs00201046_m1) and hyaluronidase 2 (Hs00201046_m1) in human samples and TNF $\alpha$ (0c3397716_g1), IL1 $\beta$ (0c03823250_s1), TLR2 (0c03824728_s1), TLR4 (0c03398502_m1), CD44 (Hs01075861_m1), and GAPDH (0C03823402_g1) were assayed using primers plus MGB TaqMan probes from Invitrogen.

\section{Results}

\section{IVH does not affect total HA levels in preterm rabbit pups and human infants}

To compare HA levels between premature rabbit pups with and without IVH, we labeled coronal sections from the forebrain with hyaluronan-binding protein (HABP). We found that HA was expressed throughout the gray matter, $\mathrm{WM}$, and periventricular ganglionic eminence (GE) and that the HA expression (HABP staining) was comparable in these regions between pups with and without IVH at postnatal days 3, 7, and 14 (Fig. 1A). Double labeling of $\mathrm{HABP}$ with $\mathrm{O} 4$ and GFAP antibodies revealed that $\mathrm{HA}$ was predominantly expressed in the extracellular matrix, and its expression was weak to absent on $\mathrm{O}^{+}$OPCs (Fig. 1A). There were focal areas of colocalization between GFAP and HA, consistent with the fact that astrocytes produce HA. We next quantified HA by fluorophore-assisted carbohydrate electrophoresis on rabbit forebrain homogenates, which confirmed that HA levels were similar between pups with IVH and without IVH (glycerol treated) at days 3, 7, and 14 (Fig. 1B). Importantly, intracerebroventricular (ICV) hyaluronidase treatment almost totally eliminated HABP binding in immunolabeled sections (Fig. 1A) and reduced HA levels by more than 10 -fold in forebrain homogenates (Fig. 1B). Comparable HA levels between rabbits with and without IVH and its marked reduction by hyaluronidase treatment were further validated by ELISA (Fig. 1C).

We next evaluated HA expression in the forebrain of premature infants (23-27 gw) with and without IVH (Table 1). Consistent with premature rabbits, HA expression in the GE and the adjacent WM was comparable between human infants with and without IVH (Fig. 1D). HA expression was almost absent on $\mathrm{MAP}_{2}{ }^{+}$neurons. However, HA was expressed by $\mathrm{O}^{+}{ }^{+} \mathrm{OPCs}$ and a few $\mathrm{GFAP}^{+}$astrocytes. Quantification of HA in homogenates from the GE and periventricular WM showed a trend toward decrease only in the GEs of infants with IVH compared to controls without IVH (Fig. 1E). However, the difference was not statistically significant.

To determine HA size, we purified the HA isolated from the rabbit brains and analyzed the extract by agarose gel electrophoresis. The resulting blue staining in agarose gel electrophoresis consisted of HA of molecular weight ranging from $50-2500 \mathrm{kDa}$ 

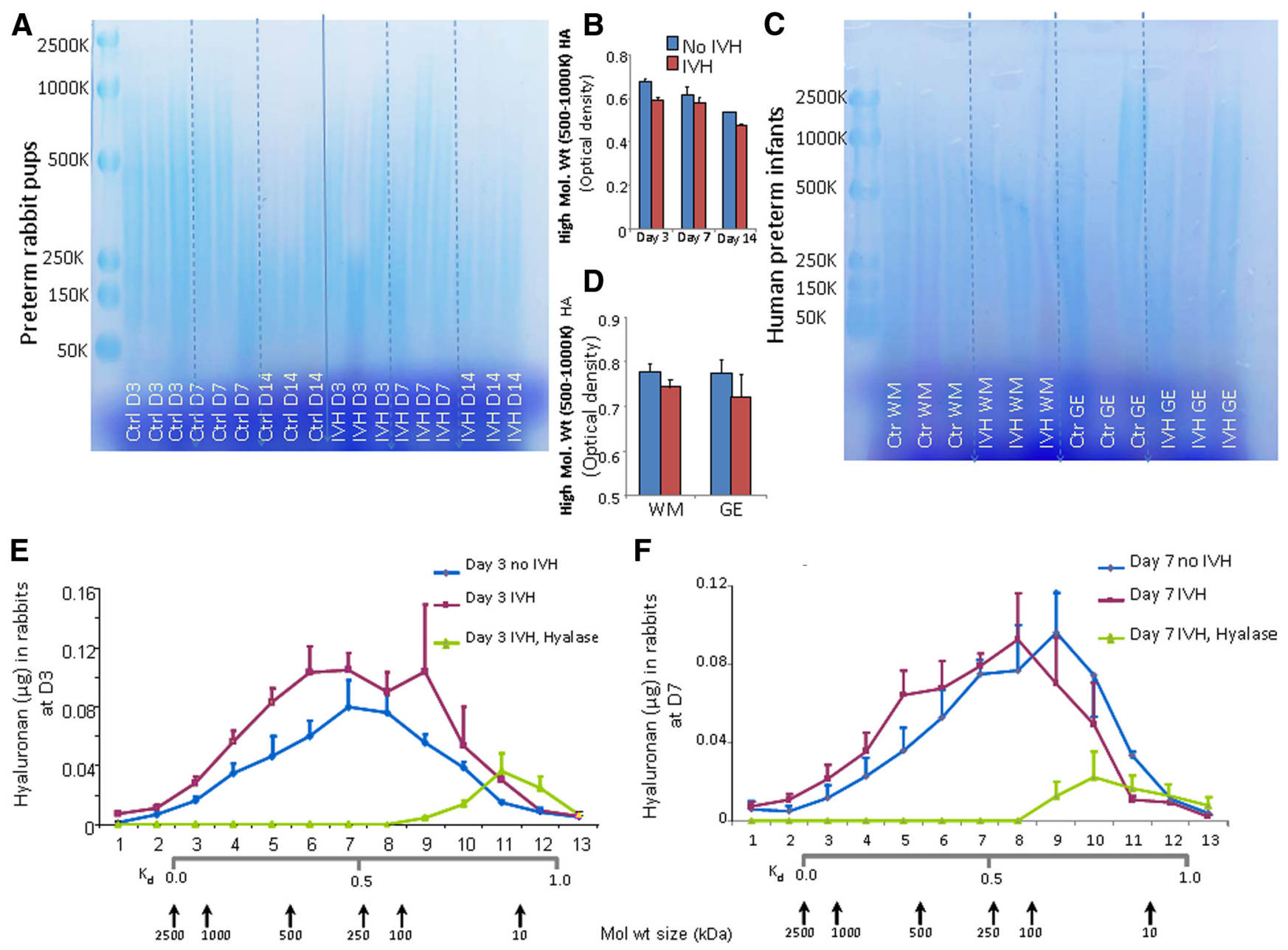

$\mathbf{F}$

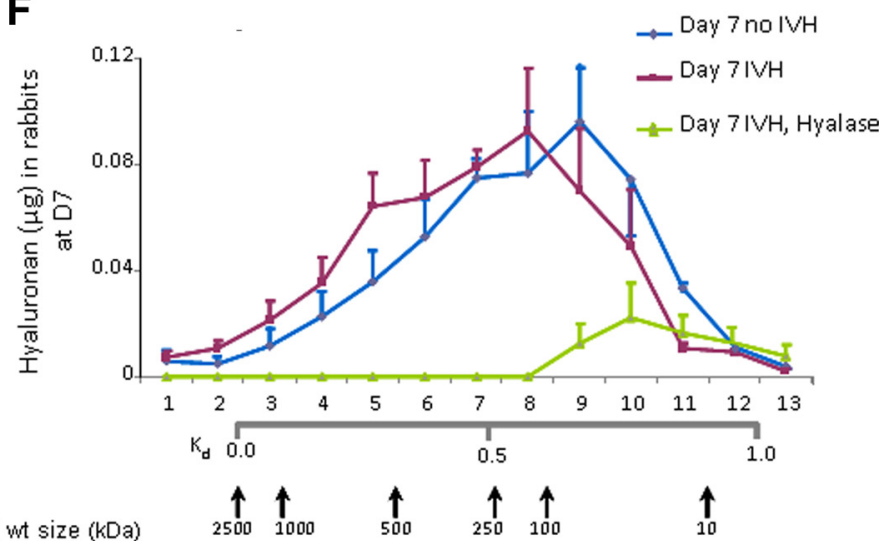

Figure 2. HA size analysis by agarose gel electrophoresis and size exclusion chromatography. $\boldsymbol{A}, \boldsymbol{B}$, Agarose gel electrophoresis shows $\mathrm{HA}$ fragments $(50-2500 \mathrm{kDa})$ in rabbit pup brain homogenates. HMW HA fragments $(500-1000 \mathrm{kDa}$ ) are comparable between pups with and without IVH compared to controls without IVH at days 3, 7, and 14. Boxed area shows HMW HA fragments of 500-1000 kDa at day 3. C, D, Agarose gel electrophoresis was performed on brain homogenates from premature infant after digestion with hyaluronidase. HMW HA fragments $(500-1000 \mathrm{kDa}$ ) are similar between infants with and without IVH in both white matter and GE. The bar charts shows mean \pm SEM ( $n=5$ each group). $E, F$, Data are mean \pm SEM ( $n=3$ pups each group). HA size was evaluated by size exclusion chromatography on samples from each of three groups of pups as indicated. Calibration was accomplished by running $2500,1000,500,250,100$, and $10 \mathrm{kDa}$ Select-HA standards. Comparisons between pups with and without IVH were insignificant at both days 3 and 7.

(Fig. 2A). Optical density measurements revealed that $500-1000$ $\mathrm{kDa}$ fragments showed a trend toward reduction in pups with IVH compared to glycerol-treated controls without IVH at day 3 (Fig. 2B). However, the comparison for HA fragments was not statistically significant between pups with and without IVH at days 3,7 , and 14 . To validate the findings of agarose gel electrophoresis, we performed size exclusion chromatography at days 3 and 7, which also showed comparable levels of HA fragments between pups with and without IVH at both days 3 and 7 (Fig. $2 E, F)$. In human infants, the distribution of HA molecular weight fragments, assessed by agarose gel electrophoresis, were comparable between neonates with and without IVH (Fig. 2C,D). Together, the data reveal that the onset of IVH does not affect total HA levels in rabbits or humans compared to controls without IVH.

\section{HAS2 is elevated in both humans and rabbits with IVH, but not HAS1 and HAS3}

To study the dynamics of HA synthesis and degradation, we evaluated expression of HAS1, HAS2, and HAS3 as well as hyaluronidase enzymes in both preterm humans (23-27 gw) and rabbits (E29) with and without IVH. Immunolabeling of forebrain sec- tions showed that HAS2 expression was more abundant in periventricular GEs of infants with IVH compared to controls without IVH (Fig. 3A). However, HAS1 and HAS3 expression was comparable between preterm infants with and without IVH. Double labeling revealed that all three synthases were expressed abundantly on $\mathrm{GFAP}^{+}$astrocytes, and weakly on doublecortinpositive $\left(\mathrm{DCX}^{+}\right)$neurons and Olig2 ${ }^{+}$OPCs (HAS3 data not shown). Western blot analyses confirmed that HAS2 levels were higher in the GEs of preterm infants with IVH compared to controls without IVH $(p=0.003$; Fig. $3 B)$, but not in the cortex or WM. HAS1 and HAS3 expressions were comparable between infants with and without IVH.

In rabbits, both mRNA and protein expression of HAS1 and HAS3 in the forebrain were comparable between pups with and without IVH at days 3 and 7 (Fig. $4 A-E$; data on rabbit HAS3 protein levels not shown). While HAS2 mRNA accumulation was lower in pups with IVH compared to controls at day 3 ( $p<0.03$, not at day 7), protein levels were higher in the forebrain of pups with IVH relative to pups without IVH at both days 3 and 7 ( $p=$ 0.003 and 0.001 , respectively; Fig. $4 C, D$ ). This difference might be attributed to alteration in the post-translational modifications of HAS2 enzymes. 

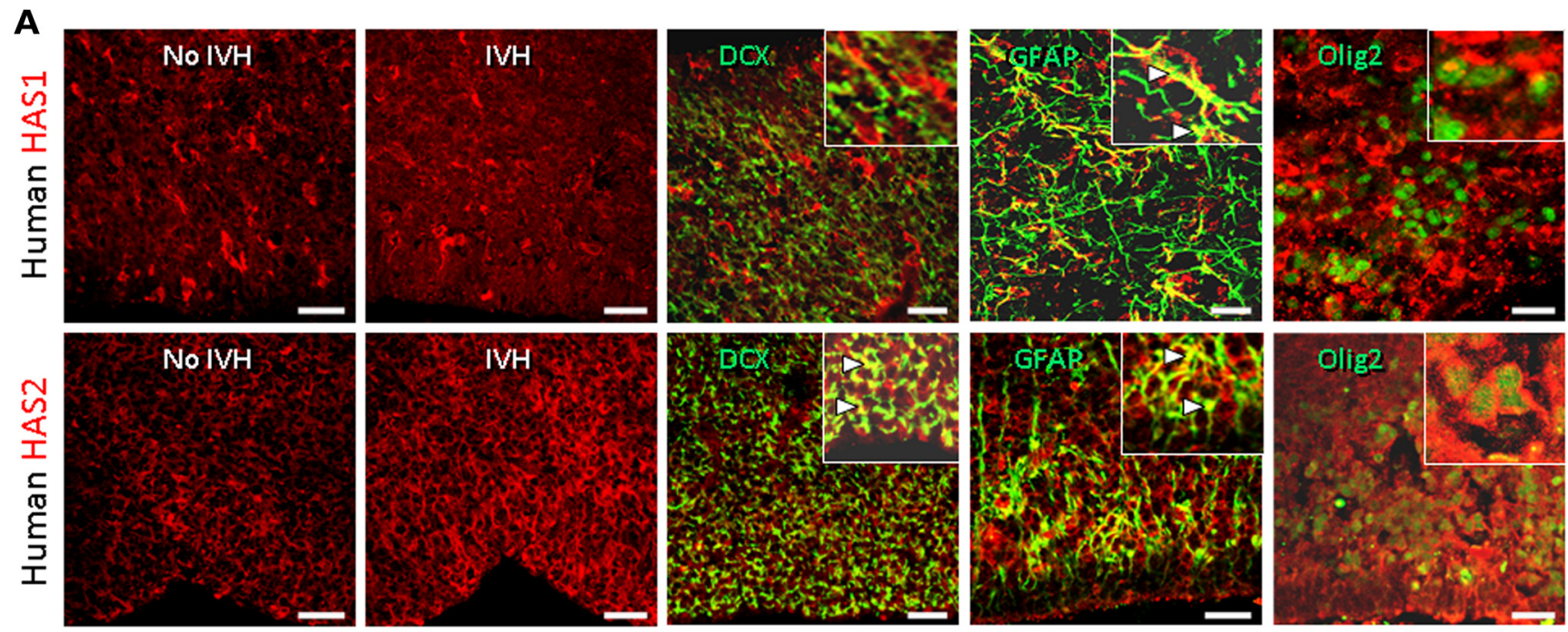

\section{B}
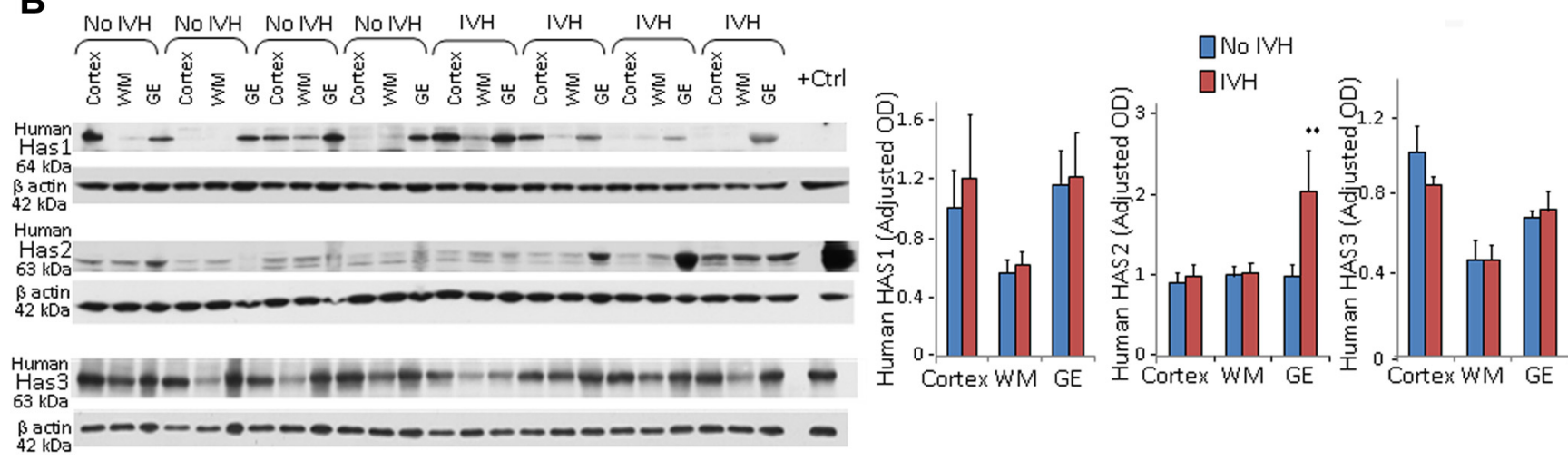

Figure 3. HAS2 enzyme is upregulated in humans with IVH. $A$, Representative immunofluorescence of cryosections from 23 gw infant labeled with HAS1 (top) and HAS2 (bottom). Insets show high-magnification images. HAS2 immunoreactivity was strong in ganglionic eminence of infants with IVH and weak in infants without IVH. However, HAS1 was similarly expressed in infants with and without IVH. Note HAS1 and HAS2 are expressed on GFAP ${ }^{+}$astrocytes (arrowheads) and a few $04^{+}$OPCs. HAS1 immunoreactivities were weak to absent on DCX ${ }^{+}$neurons, but HAS2 was expressed on $D C X^{+}$neurons (arrowheads). Scale bars: $20 \mu \mathrm{m}$. B, Western blot analyses were performed for HAS1, HAS2, and HAS3 on homogenates made from tissues taken from cortical plate (cortex), embryonic WM, and GEs of preterm infants with and without IVH, as indicated. The bar charts show mean \pm SEM ( $n=6$ each). Values were normalized to $\beta$ actin levels. HAS2 levels were elevated in the ganglionic eminence of infants with IVH compared to controls without IVH, but not HAS1 or HAS3. ${ }^{* *} p<0.01$ (no IVH vs IVH in the ganglionic eminence).

RT-qPCR data revealed that hyaluronidase-1 (Hyal-1) and Hyal-2 mRNA expressions were similar between human infants with and without IVH (Fig. 4F,G). PH20 mRNA expression could not be detected in human brains with commercially available two TaqMan primers (Invitrogen). Together, HAS2 enzymes are elevated in both rabbits and humans with IVH.

\section{CD44, TLR2, and TLR4 are elevated in the GEs of infants with IVH}

Since HA fragments signal responses through CD44 and the TLR2/4 receptors (Jiang et al., 2011), we assayed their expression in the forebrain of premature infants with and without IVH. Immunolabeling of forebrain sections demonstrated that CD44 expression was more abundant in the GEs of premature infants with IVH compared to controls without IVH (Fig. 5A). In addition, CD44 immunoreactivity was significantly higher in the GE compared with the embryonic WM and neocortex of infants both with and without IVH. Double immunolabeling revealed that $\mathrm{CD} 44$ was expressed on $\mathrm{GFAP}^{+}$astrocytes, $\mathrm{O}^{+}$OPCs, and $\mathrm{Iba}^{+}{ }^{+}$microglia, but not on $\mathrm{DCX}{ }^{+}$neurons. Western blot analyses confirmed that CD44 levels were elevated in the GEs of in- fants with IVH relative to controls without IVH ( $p<0.001$ ), but not in the neocortex or WM (Fig. 5B).

We next assessed TLR2 expression in premature infants with IVH compared to controls without IVH. We noted higher immunoreactivity of TLR2 in the GEs of premature infants with IVH than in controls without IVH (Fig. 5A). TLR2 was expressed on several $\mathrm{Ibal}^{+}$microglia and a few $\mathrm{GFAP}^{+}$astrocytes, but not on Olig2 ${ }^{+}$OPCs or $\mathrm{DCX}^{+}$neurons. Consistent with immunohistochemistry, Western blot analyses showed higher levels of TLR2 in the GEs of premature infants with IVH compared to controls without IVH ( $p<0.011$; Fig. $5 B)$.

Similar to TLR2 expression, TLR4 immunoreactivity was more abundant in the periventricular GEs of infants with IVH compared to controls without IVH. TLR4 was expressed on $\mathrm{GFAP}^{+}$astrocytes, $\mathrm{DCX}^{+}$neurons, and several O4${ }^{+}$OPCs, but not on $\mathrm{Iba}^{+}{ }^{+}$microglia (Fig. 5A). TLR4 protein quantification by Western blot analyses revealed that TLR4 expression was elevated in the GEs of human infants with IVH compared to controls ( $p=$ 0.02 ; Fig. $5 B$ ), but not in the WM or cortex. Together, IVH results in higher expression of HA receptors-CD44, and TLR2/4-in the periventricular GE. Since all OPCs originate from GE prena- 
A
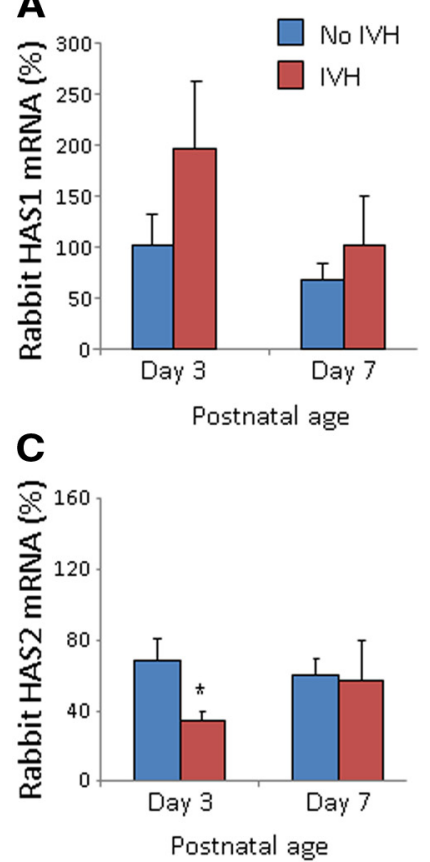

E

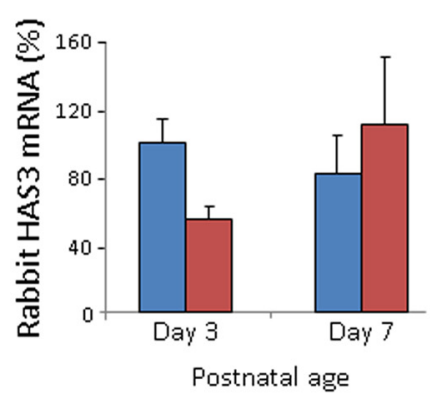

B

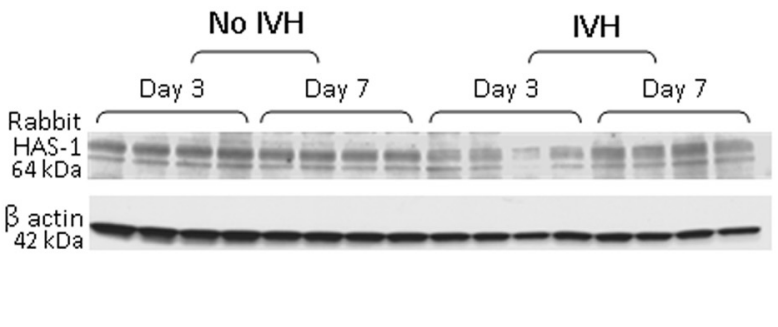

D

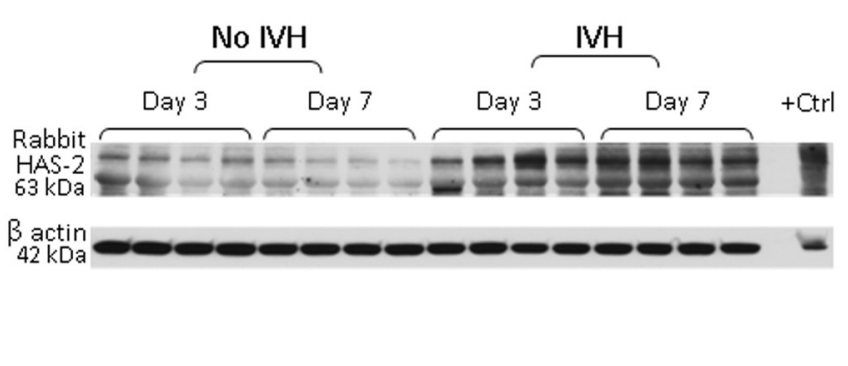

F

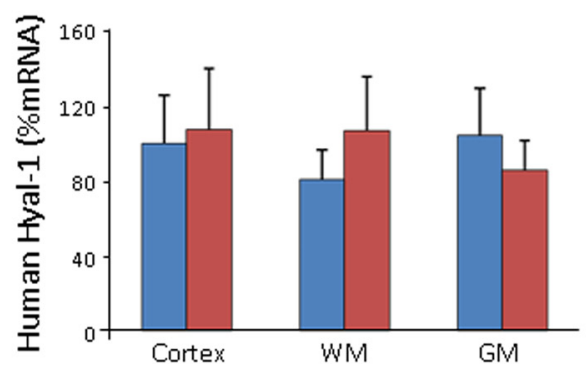

G

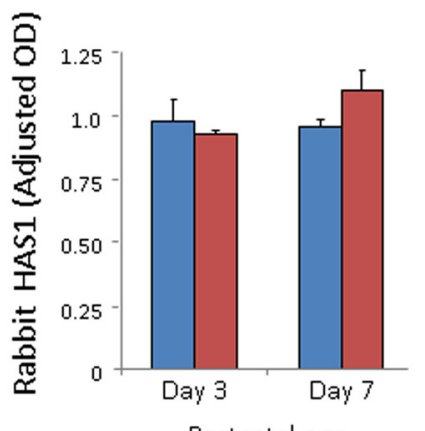

Postnatal age

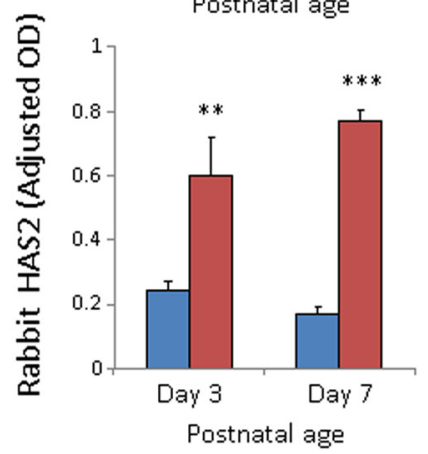

Figure 4. HAS2 expression higher in rabbits with IVH. $\boldsymbol{A}, \boldsymbol{B}, \mathrm{HAS} 1 \mathrm{mRNA}$ expression was comparable between rabbit kits with and without IVH at both days 3 and 7 . Western blot analyses for HAS1 was performed on lysates made from forebrain slices of rabbit kits with and without IVH, as indicated. HAS1 protein levels were comparable between kits with and without IVH. $\boldsymbol{C}, \boldsymbol{D}$, HAS2 mRNA expression was less in rabbit kits with IVH compared to controls at day 3, but not at day 7 . Western blot analyses for HAS2 were performed on lysates made from forebrain slices of rabbit kits with and without IVH, as indicated. Note that HAS2 levels are elevated in kits with IVH relative to controls without IVH at both days 3 and $7 .{ }^{*} p<0.05$; ${ }^{* *} p<0.01$; ${ }^{* * *} p<0.001$ (for IVH vs no IVH). $\boldsymbol{E}$, HAS3 mRNA was similar between kits with and without IVH. F, G, Human Hyal-1 and Hyal-2 mRNA expressions were similar between infants with and without IVH at both days 3 and 7. The bar charts shows mean $\pm \operatorname{SEM}(n=5$ each group).

tally to populate the white matter (Kessaris et al., 2006), elevated expressions of CD44 and TLR2/4 in the GE is likely to impact production and maturation of OPCs as well as myelination of the adjacent white matter.

\section{Hyaluronidase treatment restores myelination}

Because HA inhibits maturation of OPCs and myelination in cell culture experiments and models of demyelination (Back et al., 2005; Sloane et al., 2010), we compared myelination among three groups of pups at day 14: (1) pups without IVH (glycerol treated), (2) vehicle-treated pups with IVH (glycerol treated), and (3) hyaluronidase-treated pups with IVH (glycerol treated). To clarify, intraperitoneal glycerol was used to induce IVH, and ICV vehicle (saline) was administered for comparison with ICV hyaluronidase. The severity of IVH was comparable between vehicleand hyaluronidase-treated groups (ventricular volume, $192 \pm 37$ $\mathrm{mm}^{3}$ vs $189 \pm 36 \mathrm{~mm}^{3}$ for vehicle and hyalase, respectively), as measured by head ultrasound. Stereological analyses of MBP in immunolabeled sections demonstrated that the volume fractions (myelin load) of MBP in the corpus callosum and corona radiata were significantly reduced in pups with IVH relative to controls without IVH $(p<0.004)$ and that ICV hyaluronidase injection restored the expression of MBP ( $p=0.049$; Fig. 6A). Accordingly, Western blot analyses verified that MBP and MAG levels were diminished in pups with IVH compared to controls without IVH $(p<0.001$ both $)$ and that hyaluronidase treatment significantly increased MBP and MAG expression in pups with IVH (both $p=0.001$; Fig. $6 B, C$ ).

Ultrastructural evaluation of the corpus callosum and corona radiata revealed that myelinated axons were fewer in pups with IVH compared to controls without IVH $(p<0.05$; Fig. $6 D)$ and that hyaluronidase treatment significantly amplified the number of myelinated axons in pups with IVH $(p<0.05)$. Moreover, the g ratio was comparable in the three groups of pups $(0.769 \pm 0.01$ vs $0.76 \pm 0.002$ vs $0.758 \pm 0.012$, in pups without IVH with IVH and hyaluronidase treatment, respectively). This suggests that hyaluronidase treatment enhances myelination and restores morphology of the myelin sheath.

Since myelination is abundant at day 14 in our animal model, we selected two epochs — day 7 and day 14-to assess progress in myelination in the three sets of rabbit pups. We found that MBP and MAG expressions were $\sim 10$-fold less at day 7 relative to day 


\section{A}
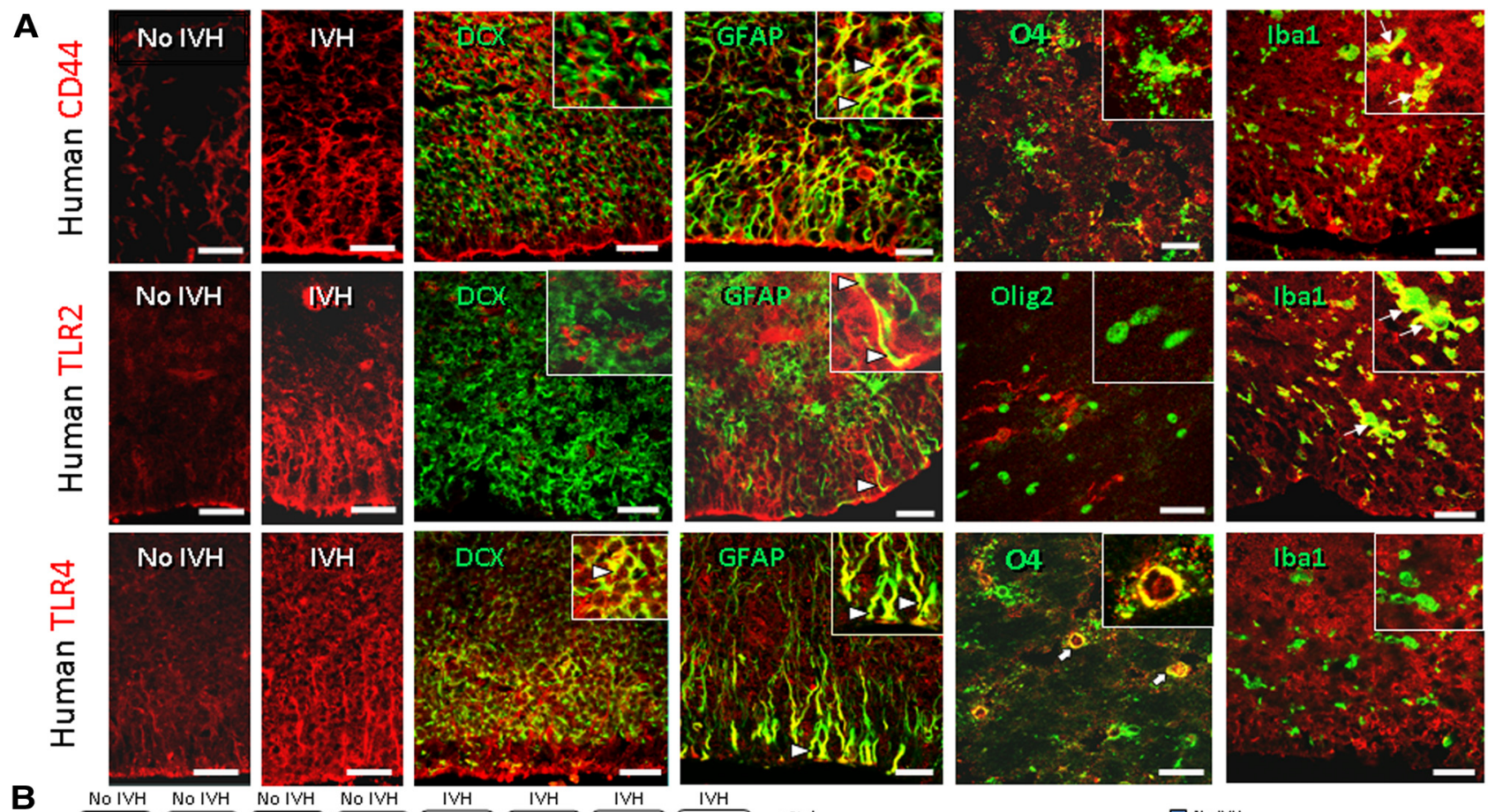

$\square$ NaIVH

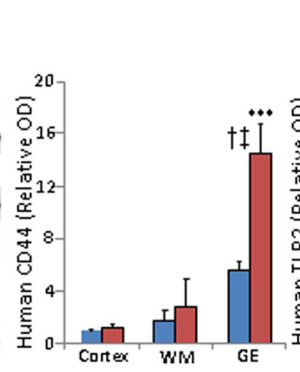

$\square \mathrm{IYH}$
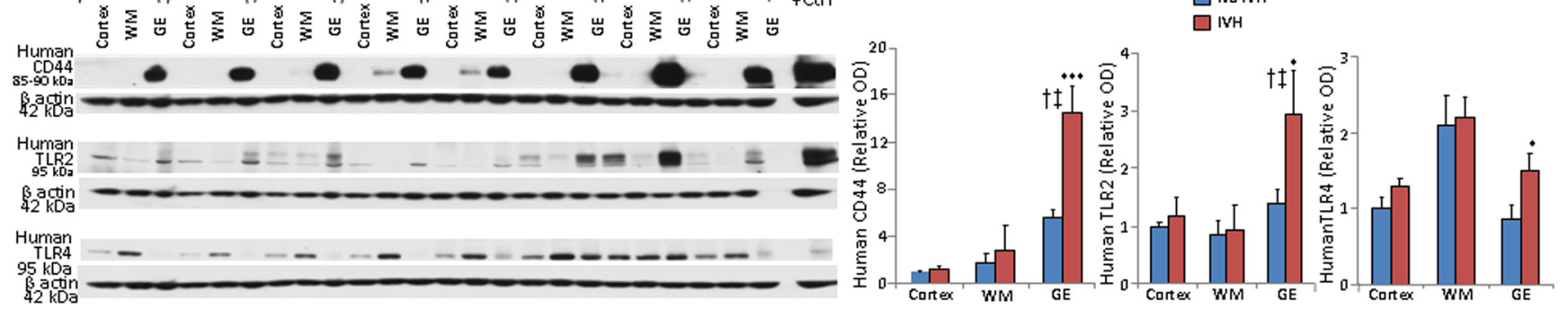

Figure 5. CD44, TLR2, and TLR4 are elevated in humans with IVH. A, Typical immunolabeling of cryosections from 23 gw infants with CD44- (top), TLR2- (middle), and TLR4-specific (bottom) antibodies. Insets show high-magnification images. Note that all the three receptors show stronger and more widespread immunoreactivity in infants with IVH relative to controls without IVH. Note that CD44 and TLR2 were expressed on GFAP ${ }^{+}$astrocytes (arrowheads) and Iba ${ }^{+}{ }^{+}$microglia (arrows), but not on OPC or DCX ${ }^{+}$neurons. TLR4 were expressed on DCX ${ }^{+}$neurons, astrocytes (arrowheads), and a few OPC (block arrows). Scale bars: $20 \mu \mathrm{m}$. B, Representative Western blot analyses performed on tissues from cortical plate, embryonic WM, and GEs of preterm infants with and without IVH using CD44- (85-95 kDa) and TLR2- and TLR4-specific ( $90 \mathrm{kDa})$ antibodies, as indicated. The bar charts show mean \pm SEM ( $n=6$ each). Values were normalized to $\beta$ actin levels. All three receptors were elevated in GEs of infants with IVH compared to controls without IVH. Both CD44 and TLR2 were elevated in the GE relative to the neocortex and white matter among infants with IVH, but not infants without IVH. ${ }^{*} p<0.05,{ }^{* * *} p<0.001$ (no IVH vs IVH in the GE); ${ }^{\dagger} p<0.01$ (GM vs WM in pups with IVH); ${ }^{\ddagger} p<0.01$ (GE vs cortex in pups with IVH).

14 in control pups without IVH and hyaluronidase-treated pups. More importantly, MBP expression was comparable between three groups-pups without IVH and vehicle- and hyaluronidase-treated pups with IVH — at day 7 on both immunohistochemistry with stereological quantification and Western blot analyses (Fig. 6E). This suggested that the process of myelination was predominant during days 7-14, and arrested myelination in pups with IVH was restored by hyaluronidase treatment during this period.

\section{Hyaluronidase treatment does not affect astrogliosis}

HA accumulates in the CNS lesions, limits gliosis, and regulates glia scar formation (Struve et al., 2005; Cargill et al., 2012). Therefore, we hypothesized that hyaluronidase treatment might reduce astrogliosis in rabbit pups with IVH. However, GFAP expression was comparable between vehicle- and hyaluronidase-treated pups with IVH in the corpus callosum and corona radiata on both stereological quantification of immuolabeled sections and Western blot analyses (Fig. 7A,B).

Since hyaluronidase treatment enhanced myelination in pups with IVH, we reasoned that this might affect myelination or gli- osis in healthy pups. To this end, we compared MBP and GFAP levels in the vehicle- and hyaluronidase-treated healthy pups at day 14 (data not shown). We found that hyaluronidase treatment did not significantly affect myelination or astrogliosis in healthy pups without IVH.

ICV hyaluronidase promotes maturation of OPC and enhances transcription of Olig1, Olig2, and MBP genes Since HA inhibits OPC maturation (Back et al., 2005; Sloane et al., 2010), we postulated that hyaluronidase treatment might affect proliferation and maturation of OPCs. To assess proliferation of OPCs in the corona radiata and corpus callosum, we compared (1) pups without IVH (glycerol treated), (2) vehicle-treated pups with IVH, and (3) hyaluronidase-treated pups with IVH for cycling $\left(\mathrm{Ki}_{6}{ }^{+}\right)$and total OPCs$\operatorname{PDGFR} \alpha^{+}$and Olig $2^{+}$- at day 3. Severity of IVH was similar between vehicle- and hyaluronidase-treated groups (ventricular volume, $197 \pm 38 \mathrm{~mm}^{3}$ vs $190 \pm 31 \mathrm{~mm}^{3}$ for vehicle- and hyalase-treated pups, respectively). The densities of total PDGFR $\alpha^{+}$cells were similar between the three sets of pups (Fig. 8A). However, the cycling PDGFR $\alpha^{+}$cells were reduced 
A
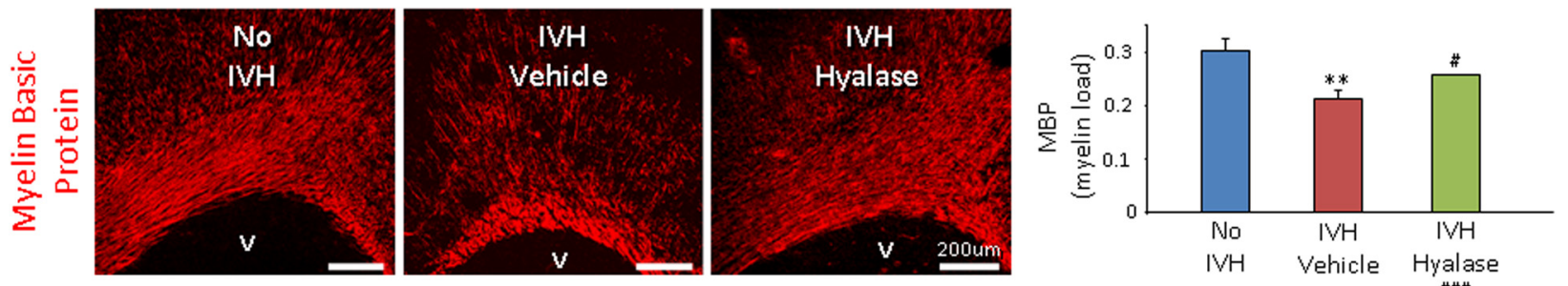

B

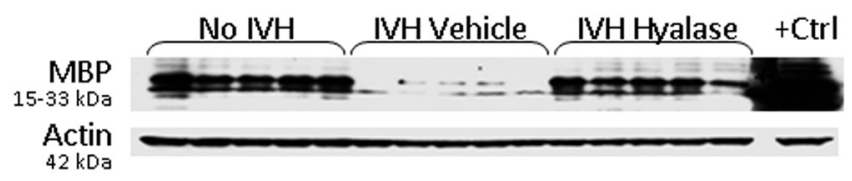

C
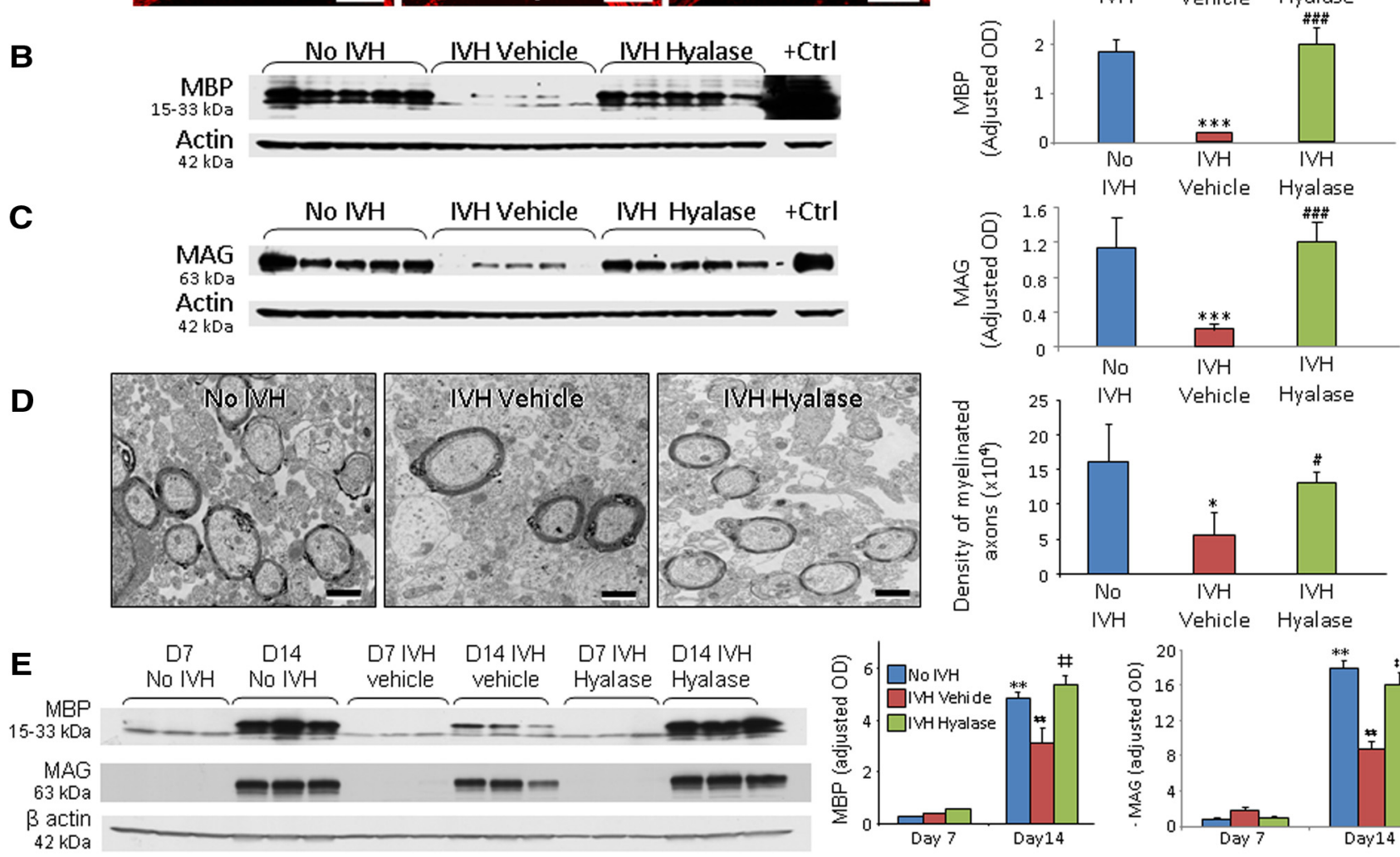

Figure 6. Hyaluronidase treatment restores myelination in rabbits with IVH. $\boldsymbol{A}$, Representative immunofluorescence of MBP in the corona radiata of day 14 pups. Volume fractions of MBP were higher in the corpus callosum and corona radiata of hyaluronidase-treated pups compared with vehicle-treated controls with IVH. V, Ventricular side. $\boldsymbol{B}$, Typical Western blot analysis for MBP in the forebrain of premature rabbit pups, as indicated, at day 14 . Adult rat brain was used as a positive control. Each lane represents a lysate from a whole coronal slice taken at the level of midseptal nucleus of one brain. MBP expression was higher in hyaluronidase-treated pups compared with vehicle-treated pups. C, Western blot analysis for MAG in the forebrain of pups as indicated at day 14 . Adult rat brain was used as a positive control. MAG expression was higher in hyaluronidase-treated pups compared with vehicle-treated controls. $\boldsymbol{D}$, Typical electron micrograph from rabbit pups without and with IVH, and pups with IVH treated with hyaluronidase at day 14. Note that myelinated axons were fewer in pups with IVH compared to controls without IVH and that hyaluronidase treatment significantly increased the number of myelinated axons in pups with IVH. $\boldsymbol{E}$, Representative Western blot analyses for MBP and MAG for three groups of pups (as indicated) at days 7 and 14 . Note the similar expression of MBP and MAG in the three sets of pups at day 7. MBP levels at day 14 were $\sim 10$-fold higher compared to day 7 in pups without IVH and hyaluronidase-treated pups with IVH. ${ }^{*} p<0.05$ (pups with vs without IVH), ${ }^{* *} p<0.01$ (no IVH pups day 7 vs day 14), ${ }^{* * *} p<0.001$ (pups with vs without IVH); ${ }^{*} p<0.05$ (vehicle- vs hyaluronidase-treated pups with IVH), ${ }^{\# \#} p<$

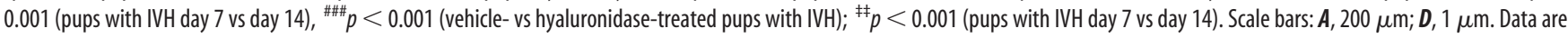
mean $\pm \operatorname{SEM}(n=5$ each group).

in pups with IVH ( $p=0.047)$ compared to controls without IVH, and hyaluronidase treatment did not affect their density. Both total and cycling Olig2 cells showed a trend toward decline in pups with IVH compared to controls without IVH at day 3 , and hyaluronidase treatment did not significantly affect their density (Fig. 8B).

We next evaluated the effect of hyaluronidase treatment on $\mathrm{OPC}$ maturation. We found that the population of $\mathrm{O}^{+} \mathrm{APC}^{+}$ cells was significantly reduced in pups with IVH relative to controls without IVH $(p<0.001)$, and hyaluronidase treatment significantly increased the density of $\mathrm{O}^{+} \mathrm{APC}^{+}$OPCs $(p<$ 0.001 ) in the corona radiata and corpus callosum (Fig. $8 C$ ). This suggests that hyaluronidase treatment restores the maturation of OPCs in pups with IVH. We also found that the levels of CNPase $\left(2^{\prime}, 3^{\prime}\right.$-cyclic nucleotide- $3^{\prime}$-phosphodiesterase), measured by Western blot analyses, were significantly reduced in pups with
IVH compared to controls $(p<0.001)$ at day 14 and that hyaluronidase treatment restored the expression of CNPase $(p=$ 0.001; Fig. $8 D$ ). Together, hyaluronidase treatment favors maturation of OPCs.

As OPC matures from the stage of specification to the terminal differentiation, Olig1, Olig2, and Sox10 transcription factors enhance maturation, whereas Id 2 and Id4 have inhibitory influences (Nicolay et al., 2007). qRT-PCR revealed that mRNA expressions of Olig2 and $M B P$ were significantly reduced in pups with IVH at day 7 ( $p=0.008$ and $<0.05$, respectively), and that hyaluronidase treatment significantly elevated levels of Olig1, Olig2, and MBP mRNA compared to vehicle controls at day 7 $(p<0.05,0.036$, and 0.048 , respectively; data not shown). Additionally, ID4 expression was elevated in pups with IVH ( $p=$ $0.036)$, and hyaluronidase treatment reduced its level $(p<$ 0.001). Together, the data show that hyaluronidase treatment 

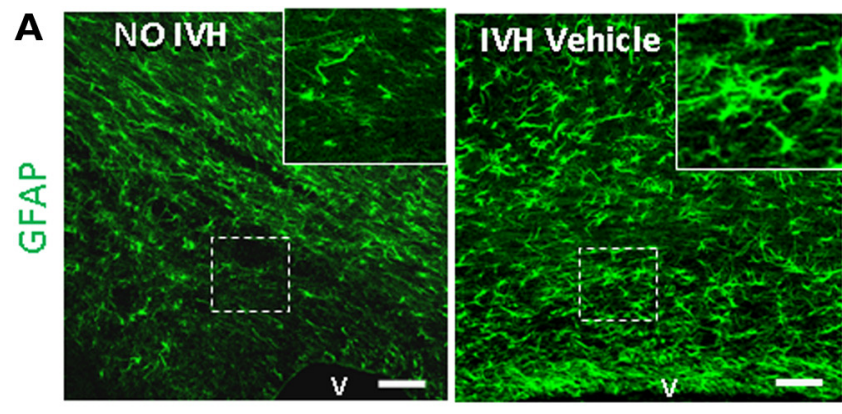

B

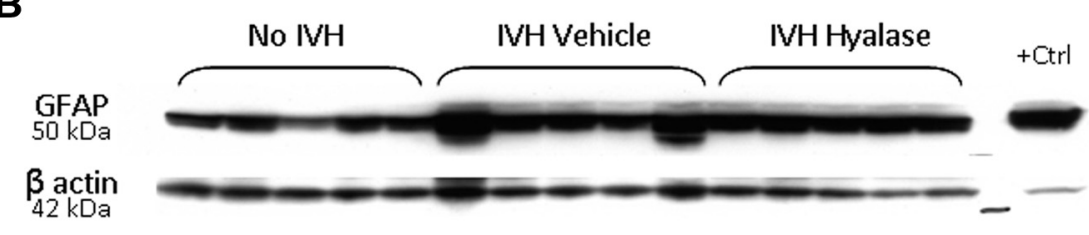

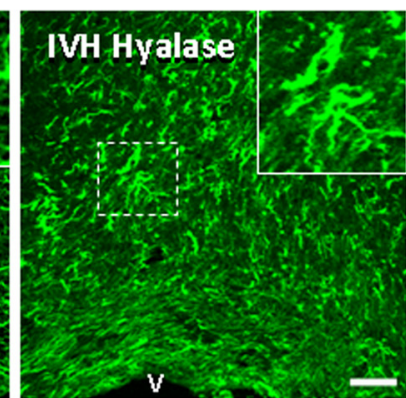
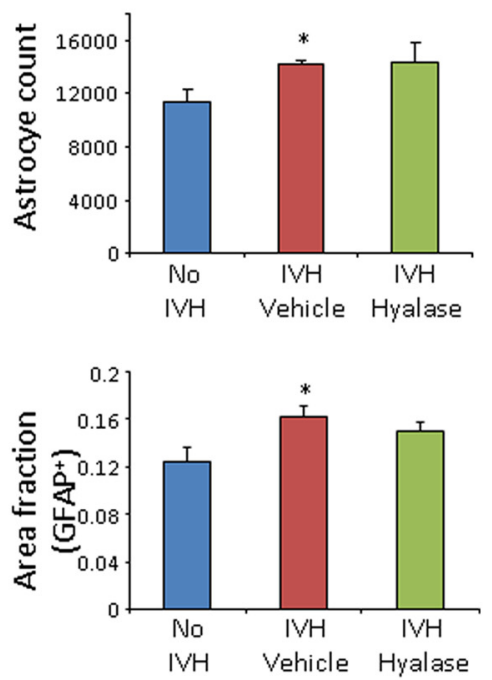
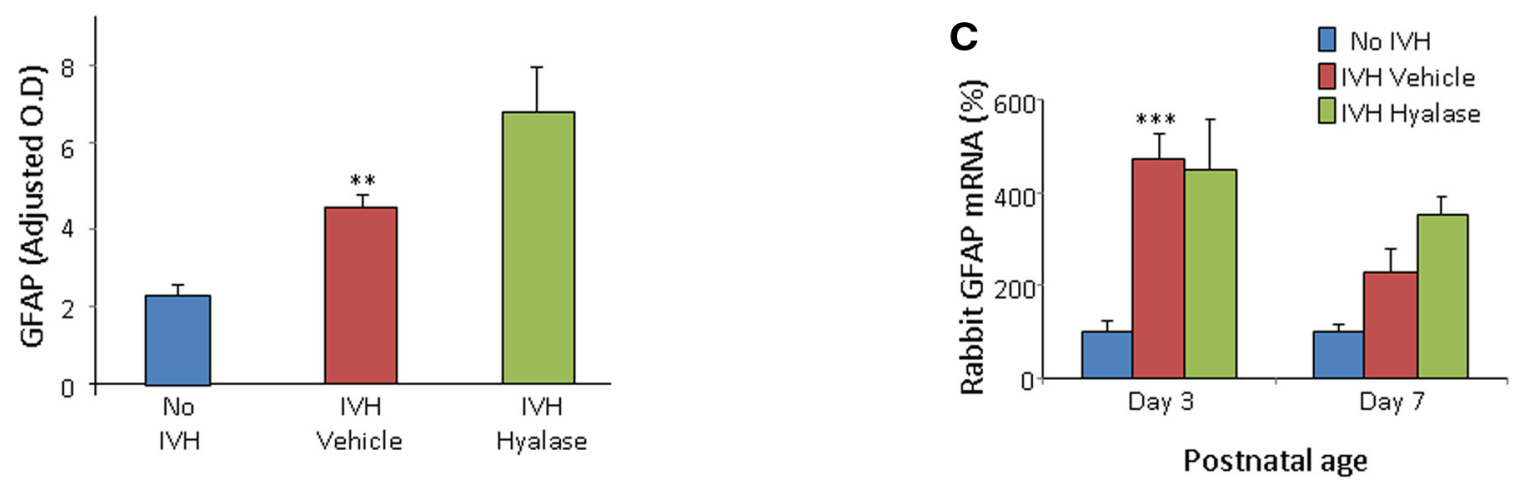

Figure 7. Hyaluronidase treatment does not alter GFAP expression. A, Representative immunofluorescence of cryosections from pups (day 14) labeled with GFAP antibody is shown, as indicated. Note abundant hypertrophic astrocytes in vehicle- and hyaluronidase-treated pups with IVH. Scale bars: $50 \mu \mathrm{m}$. V, Ventricular side. Hyaluronidase treatment did not affect volume fraction of astroglial fibers compared to vehicle controls on stereological analyses. $\boldsymbol{B}$, Western blot analyses for GFAP were performed in forebrain homogenates of pups (day 14 ). Adult rat brain was the positive control. Values were normalized to $\beta$ actin. Hyaluronidase treatment did not affect GFAP expression in pups with IVH. The graphs show mean \pm SEM $(n=5$ each). C, GFAP mRNA expression was not affected by hyaluronidase treatment. ${ }^{*} p<0.05$; $^{* *} p<0.01$; ${ }^{* * *} p<0.001$ (no IVH vs IVH).

restores expression of MBP, Olg1, Olig2, and ID4 bHLH transcription factors in pups with IVH.

\section{Hyaluronidase treatment suppresses proinflammatory \\ cytokines, microglial infiltration, and $\mathrm{HA}$ receptors}

HA and its binding receptors regulate transcription of inflammatory genes, recruitment of inflammatory cells, and release of cytokines (Petrey and de la Motte, 2014). Therefore, we assessed the effect of hyaluronidase treatment on IVH-induced inflammation. We found that the number of $\mathrm{Ibal}^{+}$and $\mathrm{CD}_{11 \mathrm{~b}}{ }^{+}$(activated) microglia were higher in pups with IVH compared to controls (glycerol treated) without IVH $(p<0.01$ and 0.001 , respectively), and that hyaluronidase treatment reduced their density $(p<0.04$ and 0.001 , respectively; Fig. $9 A, B)$.

We next quantified mRNA expression of proinflammatory cytokines, including $T N F \alpha, I L-1 \beta$, and $\gamma$ interferon, by qRTPCR (TaqMan probes). We noted that both TNF $\alpha$ and $I L-1 \beta$ expression were elevated in pups with IVH compared to controls at day 3 ( $p=0.017$ and 0.02 , respectively), but not at day 7. Importantly, hyaluronidase treatment reduced their expression $(p=0.009$ and 0.015 , respectively; Fig. 9C). $\gamma$-interferon levels were comparable between rabbits with and without IVH.

We then assayed mRNA expression of CD44 and TLR2/4 receptors by qRT-PCR (TaqMan probes). We found that CD44 gene expression was elevated in pups with IVH compared with controls at both days 3 and 7 (both $p<0.001$; Fig. $9 D)$, and hyaluronidase treatment reduced CD44 levels at both days $(p=0.006$ and 0.03 , respectively). TLR2 and TLR4 mRNA expressions were also elevated in pups with IVH relative to controls at day 3 ( $p<0.001$ and 0.002, respectively; Fig. $9 D)$, but not at day 7 . Importantly, hyaluronidase treatment significantly reduced TLR 4 expression $(p=0.005)$, but not TLR2 expression.

Consistent with qRT-PCR, immunolabeling showed that CD44 and TLR4 were more abundantly expressed in the periventricular WM of pups with IVH compared to controls without IVH, and that hyaluronidase treatment significantly reduced their immunoreactivity (data not shown). Western blot analyses confirmed that TLR4 levels were elevated in pups with IVH compared with controls at day $3(p<0.05)$, and that hyaluronidase treatment reduced the TLR4 expression ( $p=0.04$; Fig. 9E). To- 

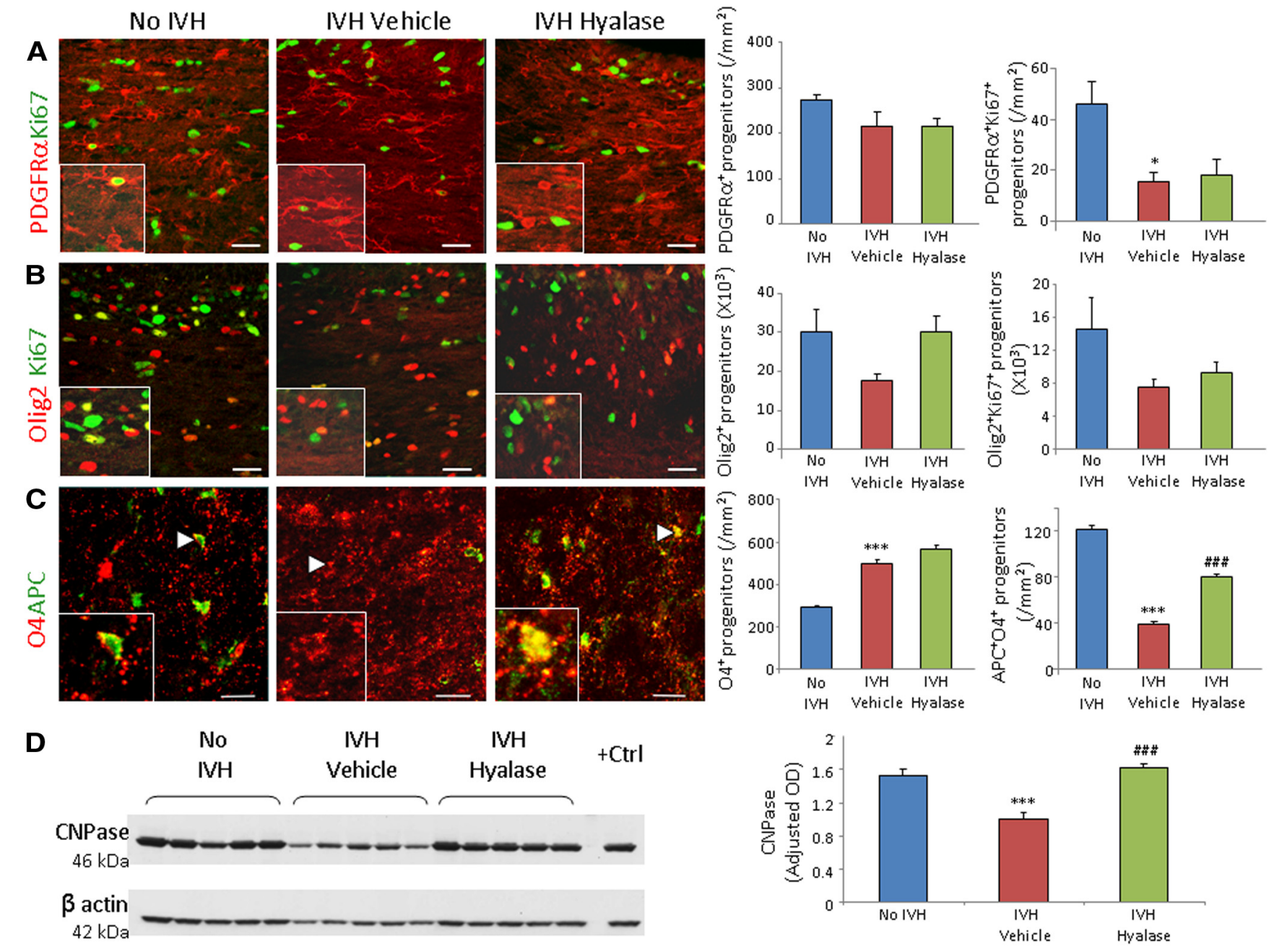

Figure 8. Hyaluronidase administration promotes OPC maturation. $\boldsymbol{A}$, Representative immunofluorescence is shown of cryosections from 3-d-old pups double labeled with PDGFR $\alpha$ - and Ki67-specific antibodies. Note that all PDGFR $\alpha^{+}$cells are comparable in the three groups as indicated; cycling PDGFR $\alpha^{+}$cells are reduced in pups with IVH, and hyaluronidase treatment does not affect these cells. $\boldsymbol{B}$, Representative immunofluorescence is shown of cryosections from 3-d-old pups double labeled with 0lig2-and Ki67-specific antibodies. Note that total and cycling 0 lig2 ${ }^{+}$cells show a trend toward reduction in pups with IVH (compared to no IVH controls) and an increase in hyaluronidase-treated pups (relative to IVH pups). C, Cryosections were double labeled with 04 (arrowhead) and APC antibodies. Note the reduced number of cells colabeled with $\mathrm{O4}^{+} \mathrm{APC}^{+}$(arrowhead) in pups with IVH compared to vehicle controls and hyaluronidase treatment increases the number in this subset of cells. Graphs show mean \pm SEM $(n=5$ each). D, Typical Western blot analyses for CNPase on the forebrain lysate of rabbit forebrain at day 14 as indicated. Note that CNPase expression is reduced in rabbits with IVH and restored after hyaluronidase treatment. ${ }^{*} p<0.05,{ }^{* * *} p<0.001$ (pups with vs without IVH); ${ }^{\# \# \#} p 0.001$ (vehicle- vs hyaluronidase-treated pups with IVH).

gether, the data show that hyaluronidase treatment reduced proinflammatory cytokines, microglial infiltration, CD44, and TLR4 expression.

Hyaluronidase administration restores neurological recovery We next performed neurobehavioral evaluations of three sets of preterm pups at day 14 (Table 3 ), as described previously (Chua et al., 2009). Vehicle- and hyaluronidase-treated groups were balanced with respect to the severity of IVH (ventricular volume, $195 \pm 27 \mathrm{~mm}^{3}$ vs $187 \pm 26 \mathrm{~mm}^{3}$ for vehicle and hyalase, respectively). We found significant weakness in the hind legs of three vehicle-treated pups, with IVH manifesting as clumsiness in the gait, whereas none of the pups in hyaluronidase-treated or healthy control group (no IVH) showed any evidence of weakness in the legs. The scores for gait were significantly higher in hyaluronidase-treated pups than in vehicle controls $(p<0.05)$. The average distance walked in $60 \mathrm{~s}$ was farther in hyaluronidase-treated pups compared with vehicle controls $(p=0.04)$. The percentage of pups showing an inability to hold their position on a ramp pitched at $60^{\circ}$ inclination for $15 \mathrm{~s}$ or longer was more in hyaluronidase-treated pups compared to saline controls (0 vs $33 \%)$. Scores for the righting reflex were significantly better in hyaluronidase-treated pups compared to vehicle-treated controls $(p<0.05)$. There was no difference in sensory and cranial nerve assessment of the three sets of rabbit pups. Importantly, we did not observe any apparent adverse effect attributable to hyaluronidase treatment among pups with IVH receiving this medication.

\section{IVH induces TSG-6 activity and synthesis of HC-HA complexes}

TSG-6 expression is upregulated in response to proinflammatory mediators, and this catalyzes the covalent transfer of HCs from $\mathrm{I} \alpha \mathrm{I}$ to HA to produce a pathological form of HA (HC-HA complex; Milner and Day, 2003; Colón et al., 2009). Since IVH induces an inflammatory response (Georgiadis et al., 2008), we evaluated autopsy samples from preterm infants with and with- 

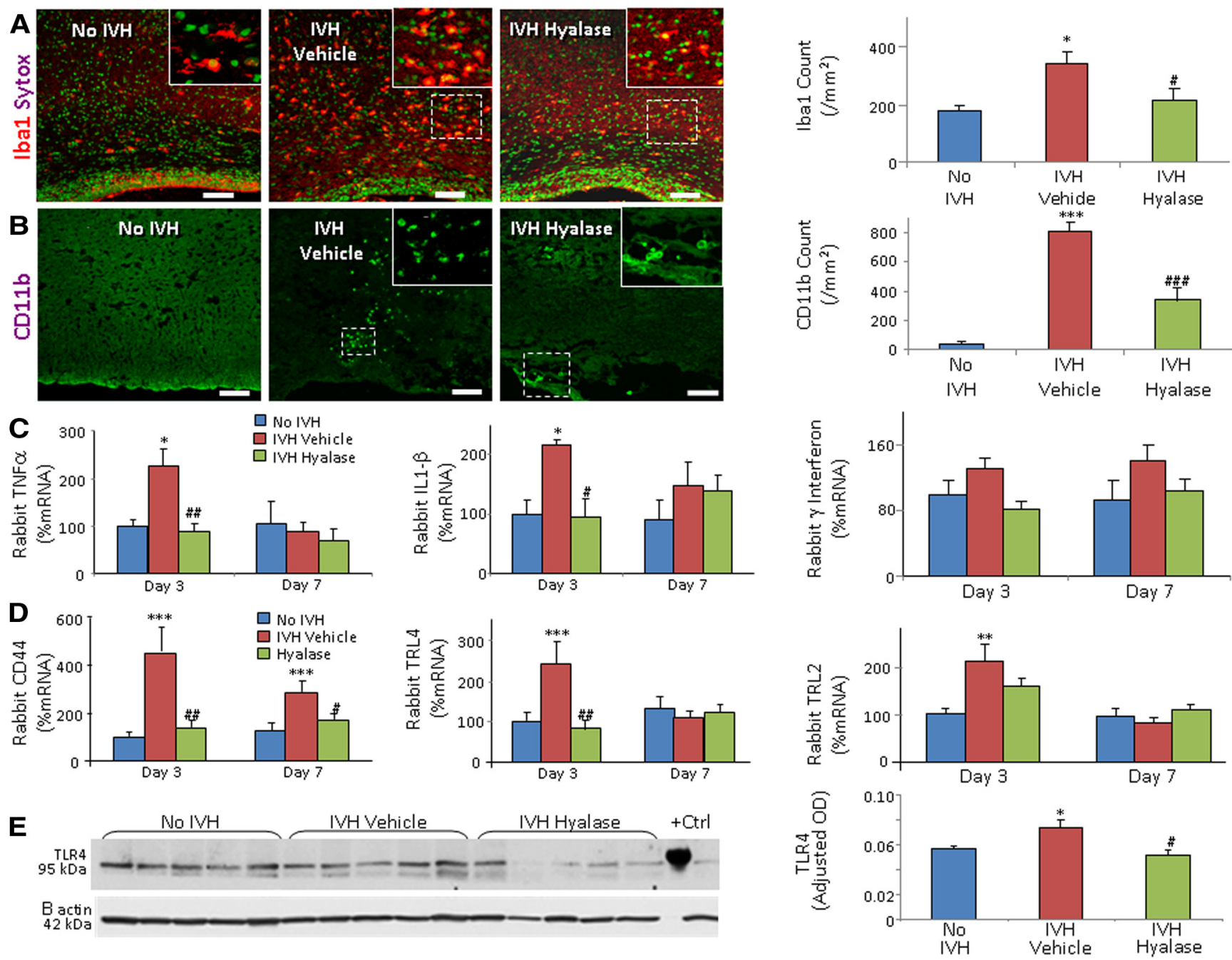

Figure 9. Hyaluronidase treatment reduced microglia density, cytokines, CD44, and TLR4 in rabbits with IVH. $\boldsymbol{A}, \boldsymbol{B}$, Representative immunofluorescence of Iba1 and CD11b are shown in the corona radiata of day 3 pups, as indicated. Sytox staining was used to label nuclei. Insets show high-magnification views of the boxed area in the image. Both lba ${ }^{+}{ }^{+}$and $C D 11 B^{+}$microglia were higher in number in pups with IVH compared to controls without IVH, and hyaluronidase treatment reduces their density. Scale bars: $50 \mu \mathrm{m}$. C, TNF $\alpha$ and IL1 $\beta$ mRNA expression were elevated in IVH compared to controls with IVH at day 3 , and hyaluronidase treatment restored the level. $\gamma$ interferon gene expression was similar in the three groups. $D$, CD44 mRNA expressions were higher in IVH compared to controls with IVH at both days 3 and 7, and hyaluronidase treatment restored the level. TLR4 gene expression was elevated in pups with IVH at day 3 , but not at P7. Hyaluronidase treatment reduced TLR4 levels. TLR2 levels were not significantly changed in the three sets of pups at days 3 and 7.E, Western blot analyses for TLR4 on the forebrain lysate of rabbit forebrain at day 3 are shown, as indicated. Note that hyaluronidase treatment reduces TLR4 protein expression in pups with IVH. Data are mean \pm SEM ( $n=5$ each group). ${ }^{*} p<0.05,{ }^{* *} p<0.01,{ }^{* * *} p<0.001$ (pups with vs without IVH); ${ }^{\#} p<0.05,{ }^{\# \#} p<0.01,{ }^{\# \#} p<0.001$ (vehicle- vs hyaluronidase-treated pups with IVH).

out IVH for HC-HA ( $85 \mathrm{kDa})$. Aliquots of forebrain homogenates were treated with hyaluronidase and incubated for $30 \mathrm{~min}$ at $4^{\circ} \mathrm{C}$, followed by $30 \mathrm{~min}$ incubation at $37^{\circ} \mathrm{C}$, to release $\mathrm{HC}$ from HC-HA complexes. Western blot analyses on these homogenates using $\mathrm{I} \alpha \mathrm{I}$ antibody showed that the $\mathrm{HC}-\mathrm{HA}(85 \mathrm{kDa})$ levels were higher in the GEs of preterm infants with IVH compared to controls without IVH ( $p=0.005$; Fig. 10A,B). HC-HA levels in the WM showed a trend toward increase in infants with IVH compared to controls.

We next evaluated endogenous TSG-6 activity in the brain extracts by adding HA oligosaccharide (14 monosaccharides). Endogenous TSG-6 activity was indicated by an appearance of a $\mathrm{HC}$ band $(85 \mathrm{kDa})$, which was formed by transfer of HCs from I $\alpha$ I onto HA oligosaccharide. Positive controls were made by addition of exogenous TSG- 6 to the brain extract, which led to formation of a strong $85 \mathrm{kDa}$ band (Fig. 10C), indicating the suitability of experimental conditions to assess TSG-6 activity. We found an increased TSG-6 activity (elevated $85 \mathrm{kDa} \mathrm{HC}$ ) in the GEs of infants with IVH compared to controls without IVH $(p=0.025$; Fig. 10C,D), but not in the WM. Together, the data demonstrate greater abundance of $\mathrm{HC}-\mathrm{HA}$ complexes and TSG- 6 activity in the GEs of infants with IVH compared to controls without IVH.

TSG-6 transfers HC from I $\alpha$ I to HA in a reversible manner. However, transfer of HC to HA oligosaccharides (8-21 oligosaccharide units) is an irreversible event. We have demonstrated the utility of this observation by irreversibly swapping HCs from HC-HA onto a HA oligosaccharide in the synovial fluid of patients with rheumatoid arthritis, thereby restoring the pathological HC-HA matrices to their normal state (Lauer et al., 2013b). Accordingly, aliquots of homogenates made from GEs of infants with IVH were incubated at $37^{\circ} \mathrm{C}$ with HA10 (10 monosaccharide units) for 24 or $72 \mathrm{~h}$. Adding exogenous recombinant TSG-6 resulted in a smear above $250 \mathrm{kDa}$ (Fig. 10, lane 4), indicating transfer of HCs from $\mathrm{I} \alpha \mathrm{I}$ to $\mathrm{HA}$, forming the HC-HA complex. Importantly, application of both HA10 and exogenous recombi- 
Table 3. Neurobehavioral evaluation of hyaluronidase- and vehicle-treated pups with IVH and controls without IVH at P14

\begin{tabular}{|c|c|c|c|c|}
\hline System & Test & $\begin{array}{l}\text { No IVH } \\
(n=12)\end{array}$ & $\begin{array}{l}\text { IVH vehicle } \\
(n=12)\end{array}$ & $\begin{array}{l}\text { IVH, hyaluronidase } \\
(n=10)\end{array}$ \\
\hline \multirow[t]{2}{*}{ Cranial nerve } & Aversive response to alcohol & $3(3,3)$ & $3(3,3)$ & $3(3,3)$ \\
\hline & Sucking and swallowing & $3(3,3)$ & $3(3,3)$ & $3(3,3)$ \\
\hline \multirow[t]{14}{*}{ Motor } & Motor activity & & & \\
\hline & Head & $3(3,3)$ & $3(3,3)$ & $3(3,3)$ \\
\hline & Fore legs & $3(3,3)$ & $3(3,3)$ & $3(3,3)$ \\
\hline & Hind legs & $3(3,3)$ & $3(2.5,3)$ & $3(3,3)$ \\
\hline & Righting reflex ${ }^{a}$ & $5(5,5)$ & $4(3,5)^{\#}$ & $5(5,5)^{* *}$ \\
\hline & ${\text { Locomotion on } 30^{\circ} \text { inclination }}^{b}$ & $3(3,3)$ & $3(2.75,3)$ & $3(3,3)$ \\
\hline & Tone': Forelimb & $0(0,0)$ & $0(0,0)$ & $0(0,0)$ \\
\hline & Tone': Hindlimb & $0(0,0)$ & $0(0,0)$ & $0(0,0)$ \\
\hline & $\begin{array}{l}\text { Inability to hold their position } \\
\text { at } 60^{\circ}\end{array}$ & $0 \%$ & $25 \%$ & $0 \%$ \\
\hline & Inclination for $15 \mathrm{~s}$ or less & & & \\
\hline & $\begin{array}{l}\text { (latency to slip down } \\
\text { the slope, if }<15 \mathrm{~s} \text { ) }\end{array}$ & & & \\
\hline & $\begin{array}{l}\text { Inability to walk more than } 60 \\
\text { inches in } 1 \mathrm{~min}(\%)\end{array}$ & $0 \%$ & $33 \%$ & $0 \%$ \\
\hline & Gait $^{d}$ & $4(4,4)$ & $3(2.5,4)^{\#}$ & $4(4,4)^{*}$ \\
\hline & Motor impairment ${ }^{e}$ & $0 \%$ & $25 \%$ & $0 \%$ \\
\hline \multirow[t]{2}{*}{ Sensory } & Facial touch & $3(3,3)$ & $3(3,3)$ & $3(3,3)$ \\
\hline & Pain & $3(3,3)$ & $3(3,3)$ & $3(3,3)$ \\
\hline
\end{tabular}

Values are the median and interquartile range. Zero is the worst response and 3 is the best response, unless noted otherwise.

${ }^{a}$ The score (range, 1-5) is the number of times the animal turned prone within $2 \mathrm{~s}$ when placed in a supine position out of five tries.

${ }^{b}$ The scoring (range, 0 -3) is as follows: 0, does not walk; 1 , takes a few steps (less than 8 inches); 2, walks for 9 -18 inches; 3 , walks very well beyond 18 inches.

'The scoring (range, 1-3) is as follows: 0 , no increase in tone; 1 , slight increase in tone; 2 , considerable increase in tone; 3 , limb rigid in flexion or extension.

${ }^{d} G$ ait was graded as 0 (no locomotion), 1 (crawls with trunk touching the ground for few steps and then rolls over) 2 (walks taking alternate steps, trunk low and cannot walk on inclined surface), 3 (walks taking alternate steps, cannot propel its body using synchronously the hind legs, but walks on $30^{\circ}$ inclined surface), 4 (walks, runs, and jumps without restriction and propels the body using synchronously the back legs, but limitation in speed, balance, and coordination, manifesting as clumsiness in gait), or 5 (normal walking).

eMotor impairment was defined as weakness in either fore or hind legs and a distance walked of less than 60 inches in $60 \mathrm{~s}$.

${ }^{*} p<0.05$; ${ }^{* *} p<0.01$ (vehicle-treated vs hyaluronidase-treated pups with IVH).

${ }^{\#} p<0.05$ (glycerol-treated pups without IVH and vehicle-treated pups with IVH).

nant TSG-6 to the brain extract (lane 5) led to an intense $85 \mathrm{kDa}$ band, which indicated swapping of the HCs from HC-HA complex onto the HA10 oligosaccharide. Collectively, use of HA10 holds promise in removal of $\mathrm{HC}$ from pathological HA in preterm infants and possibly in rabbits with IVH.

We next assessed TSG-6 mRNA expressions in preterm rabbit pups with and without IVH at days 3 and 7. We found that TSG-6 gene expression was significantly higher in pups with IVH compared to controls without IVH at P3 ( $p=0.009$; Fig. $10 G$ ), but not at P7. Accordingly, immunolabeling showed that TSG-6 was more abundantly expressed in GE and WM of rabbits with IVH compared to controls without IVH (data not shown). Together, the data suggest that TSG-6 expression is upregulated in subjects with IVH, which induces generation of $\mathrm{HC}-\mathrm{HA}$ complexes in the brain.

\section{ICV HA oligosaccharide administration reduces inflammation and promotes myelination}

Because HC-HA complexes exhibit proinflammatory activity, we postulated that removal of HCs from the HC-HA complexes might reduce inflammation and restore myelination in rabbit pups with IVH. Since HA oligosaccharides are irreversible $\mathrm{HC}$ acceptors, unlike high molecular weight HA, we compared (1) pups without IVH (glycerol treated), (2) pups vehicle treated with IVH, and (3) HA10-treated pups with IVH for microglial density and proinflammatory cytokines. Vehicle was administered intracerebroventricularly, just as HA10. We found that the densities of both $\mathrm{Iba}^{+}$and CD $11 \mathrm{~b}^{+}$ microglia in the periventricular WM were higher in the pups with IVH compared to controls without IVH at day $3(p=$ 0.01 and 0.001 , respectively), and HA oligosaccharide treatment reduced their number in pups with IVH $(p=0.015$ and 0.001 , respectively; Fig. $11 A, B)$. Accordingly, TNF $\alpha$ and $I L 1 \beta$ mRNA accumulation were higher in the pups with IVH compared to glycerol controls ( $p<0.001$ and 0.004 , respectively), and HA oligosaccharide treatment reduced their levels in pups with IVH at day 3 ( $p=0.001$ and 0.036 , respectively; Fig. $11 C)$. HA oligosaccharide also reduced TLR4 expression in pups with IVH at day $3(p<0.01)$, but not CD44 and TLR2 expression (Fig. 11D). Hence, HA oligosaccharide administration appears to alleviate inflammation by reducing microglial infiltration, proinflammatory cytokines, and TLR4 levels.

We next evaluated the effect of HA oligosaccharide treatment on myelination and astrogliosis. Stereological quantification of immunolabeled sections revealed that the volume fractions (myelin load) of MBP in the corpus callosum and corona radiata were significantly higher in HA oligosaccharide-treated pups with IVH relative to vehicle controls with IVH ( $p=0.014$; Fig. 12A). Western blot analyses demonstrated that all three-MBP, MAG, and CNPase-levels were higher in HA oligosaccharide-treated pups with IVH compared to vehicle controls with IVH (all $p<0.001$; Fig. 12B-D). Moreover, GFAP expression-measured by stereological quantification and Western blot analysis-was similar between HA oligosaccharide and vehicle-treated pups with IVH (Fig. 12E). Together, the data demonstrate that HA oligosaccharide treatment enhances myelination without significantly inhibiting astrogliosis.

\section{HA oligosaccharide treatment promotes neurological recovery}

We performed neurobehavioral evaluations on the three sets of preterm pups at day 14: (1) pups without IVH (glycerol treated), (2) vehicle-treated pups with IVH, and (3) HA oligosaccharide-treated pups with IVH (Table 4). The three groups were similar with respect to the severity of IVH (179 \pm 27 vs $172 \pm 17 \mathrm{~mm}^{3}$ for HA10 and vehicle, respectively), as quantified by head ultrasound. We found that one kit in the vehicle-treated group had weakness in all four extremities and was not able to walk at all. In addition, three pups in this group had significant weakness in the hind legs manifesting as clumsiness and asymmetry in the gait. In the HA oligosaccharidetreated pups, one pup had significant weakness in the back legs. Conversely, none of the pups in the glycerol-treated control (no IVH) group exhibited significant weakness in the fore or hind legs. The scores for gait and movement of the hind leg were significantly higher in HA oligosaccharide-treated pups than in saline controls $(p<0.05)$. The average distance walked in $60 \mathrm{~s}$ was farther in HA oligosaccharidetreated pups compared with vehicle controls (114 \pm 17 vs $89.1 \pm 12.2$ inches). There was no difference in sensory and cranial nerve assessments of the three sets of rabbit pups. Overall, HA oligosaccharide-treated pups performed superior on neurobehavioral scoring compared with vehicle controls.

\section{Discussion}

In this study, we showed that the development of IVH increases the expression of HA-associated receptors (CD44, TLR2, and 

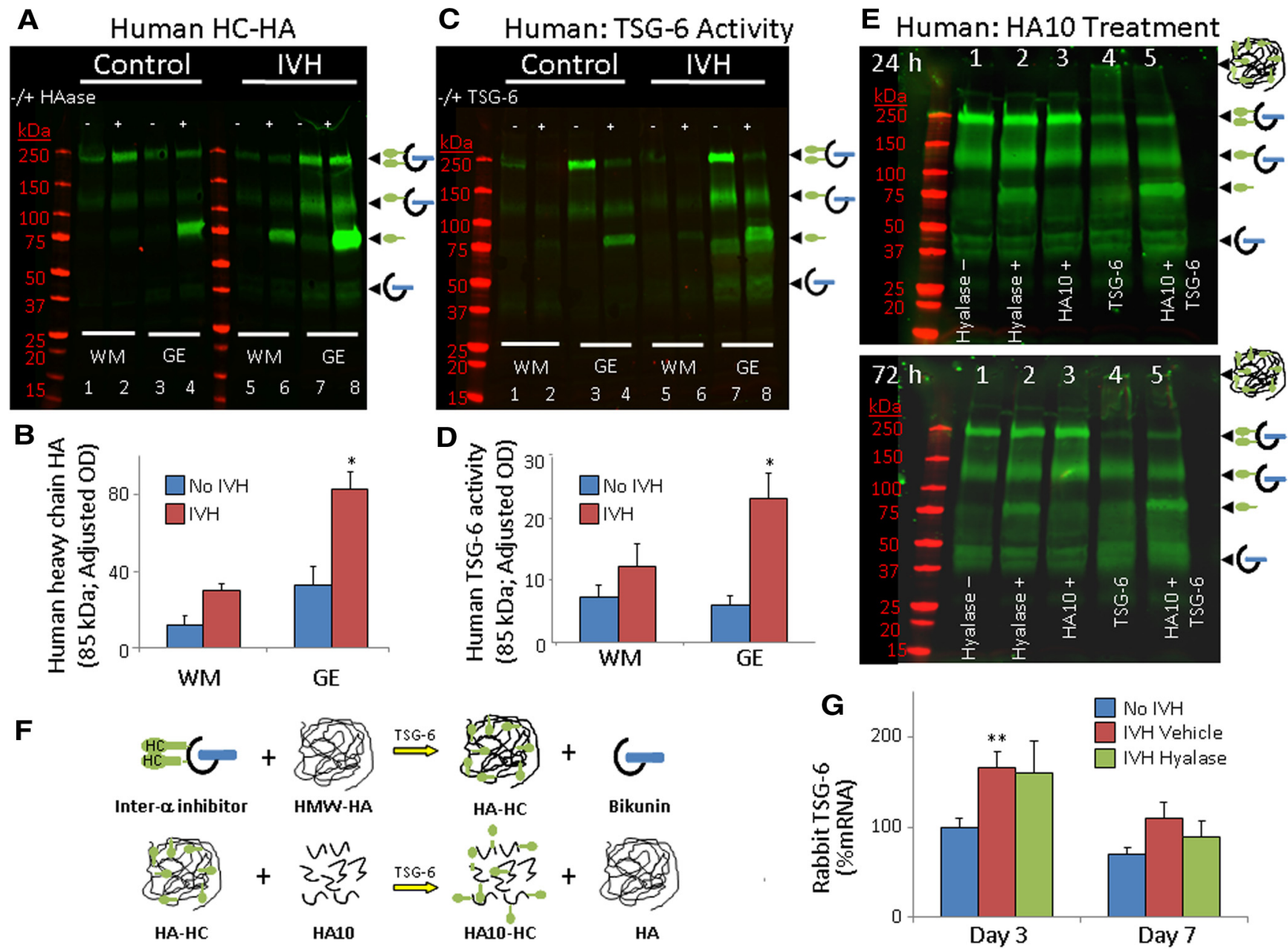

Figure 10. IVH induces TSG-6 activity and synthesis of HC-HA complexes. $A, B$, Representative Western blot analyses performed on tissues from embryonic WM and GEs of preterm infants with and without IVH using $|\alpha|$ specific antibody. Homogenates were digested with hyaluronidase and were run with undigested samples in alternate lanes. The 250, 150, and 85 kDa bands indicate $|\alpha|$, pre-l $\alpha \mathrm{l}$, and free HCs, respectively. Data are mean \pm SEM $(n=4$ each). Note that the HC-HA concentration is higher in GEs of infants with IVH compared to controls without IVH. C, $\boldsymbol{D}$, Typical Western blot analyses performed on tissues from WM and GEs of premature infants with and without IVH using I $\alpha$ antibody. HA oligosaccharide (10 monosaccharides size) was added to each homogenate in C. Some homogenates were treated with human recombinant TSG-6 as a positive control. Data are mean \pm SEM ( $n=4$ each). Note that the HC-HA concentration is higher in GES of infants with IVH compared to controls. $\boldsymbol{E}$, Representative Western blot analyses performed on tissues from GEs of preterm infants with IVH using an I $\alpha 1$-specific antibody. Homogenates were digested with hyaluronidase; treated with HA10, TSG-6, or both TSG and HA10; and incubated for 24 (top) or $72 \mathrm{~h}$ (bottom). F, Schematic showing TSG-6-mediated enzymatic transfer of HCs from $|\alpha|$ to HA, forming the HC-HA complex (top), and that these HCs can be irreversibly swapped from HC-HA onto HA10. G, Data are shown as mean \pm SEM ( $n=5$ each). TSG-6 mRNA expressions were higher in IVH compared to controls at day 3 , but not at day 7.

TLR4) and formation of HC-HA complexes, but total HA levels remain unchanged. More importantly, we demonstrated that two therapeutic strategies enhanced myelination and clinical recovery in rabbits with IVH. First, digestion of HA by ICV hyaluronidase treatment reduced CD44 and TLR4 expression, microglia infiltration, and proinflammatory cytokines, and enhanced myelination in rabbits with IVH. Second, ICV HA oligosaccharide reduced inflammation and enhanced myelination, conceivably via irreversible $\mathrm{HC}$ transfer from pathological HC-HA to the HA oligosaccharide.

The most important finding of the present study was that hyaluronidase treatment removed HA from the forebrain and restored myelination and clinical recovery in preterm rabbits with IVH. Specifically, we demonstrated that hyaluronidase treatment (1) reduced microglia infiltration, proinflammatory cytokine levels, and CD44 and TLR4 expression; (2) reversed maturational arrest of OPCs, (3) induced transcription of Olig1, Olig2, and MBP genes favoring myelination; and, thus, (4) promoted myelination and neurological recovery in a developmental rabbit model of IVH-induced WM injury. Hence, restoration of OPC maturation after hyaluronidase treatment was seemingly mediated by two interwoven signaling cascades, including reduction in levels of HA and CD44 (inflammation independent) and suppression of inflammation (inflammation dependent). Cell culture experiments have demonstrated that HA blocks OPC maturation (Marret et al., 1994; Back et al., 2005), and studies in an adult rodent model have shown that HMW HA accumulation in chronic lesions of demyelination inhibits OPC maturation (Back et al., 2005). Intriguingly, mice engineered to overexpress CD44 also exhibit inflammation-independent demyelination (Tuohy et al., 2004). Moreover, our previous study showed that suppression of inflammation by COX2 inhibition or TNF $\alpha$ downregulation promotes myelination in this animal model (Vinukonda et al., 2010). Other than in the brain, hyaluronidase PH20 also inhibits lipopolysaccharide induced neutrophil and macrophage recruitment in an air pouch model of inflammation (Huang et al., 2014). These studies reinforce our notion that hyaluronidase treatment promotes OPC maturation and myeli- 

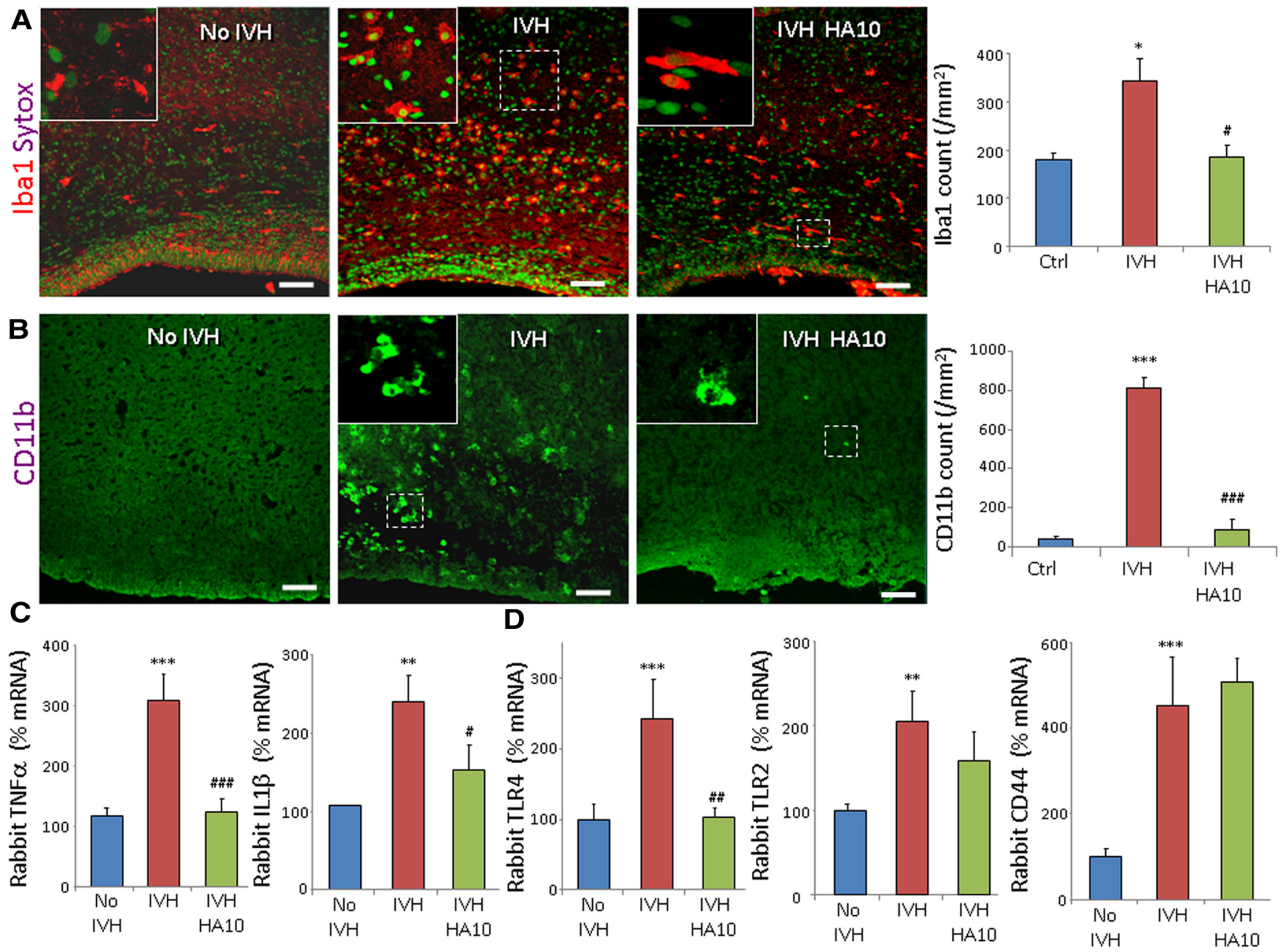

Figure 11. Intraventricular injection of an HA10 attenuates inflammation in IVH. $A, B$, Representative immunofluorescence of Iba1 and CD11b in the corona radiata of day 3 pups, as indicated. Sytox was used for nuclear staining. Insets show high-magnification views of the boxed areas in the image. Both Iba $1^{+}$and CD11 ${ }^{+}$microglia were more abundant in pups with IVH compared to controls without IVH, and HA10 treatment reduced their density. Scale bars: $50 \mu \mathrm{m}$. C, TNF $\alpha$ and IL1 $\beta$ mRNA expression was elevated in pups with IVH compared to controls with IVH at day 3 , and HA10 treatment reduced their levels. D, HA oligosaccharide treatment suppressed TLR4, but not TLR2 or CD44, at day 3. Data are mean \pm SEM $\left(n=5\right.$ each group). ${ }^{*} p<0.05$, ${ }^{* *} p<0.01,{ }^{* * *} p<$ 0.001 (pups with vs without IVH); ${ }^{\#} p<0.05,{ }^{\# \#} p<0.01,{ }^{\# \#} p<0.001$ vehicle- vs hyaluronidase-treated pups with IVH.

nation in pups with IVH by both inflammation-dependent and inflammation-independent mechanisms.

A seminal observation made in this study was an elevation in TSG-6 expression and an abundance of HC-HA complexes in the forebrains of subjects with IVH. More importantly, we found that HA10 treatment to aliquots of fore brain homogenates (autopsy samples from preterm infants) eliminated HC from pathological HC-HA complexes. Although we could not demonstrate HC-HA complexes in rabbits with IVH in the absence of a suitable antibody, HA10 treatment to remove HC suppressed inflammation and enhanced myelination as well as neurologic outcome of pups with IVH. Little is known about the function of TSG-6 activity and HC-HA complexes. TSG-6 production is induced by proinflammatory mediators including TNF $\alpha$, IL-1 $\beta$, and prostaglandin $\mathrm{E} 2$, and it catalyzes transfer of $\mathrm{HCs}$ from inter$\alpha$-inhibitor onto HA to form the pathological HC-HA complex (Milner and Day, 2003). TSG-6 is also considered to exhibit antiinflammatory and cytoprotective effects (Watanabe et al., 2013; Zhang et al., 2013; Kim et al., 2014). However, HC-HA complexes promote inflammation by causing adhesion of HA to the CD44 on the neutrophil cell surface and increase their extravasation into the interstitial tissues (Zhuo et al., 2006). An increase in
TSG-6 expression and elevated HC-HA complex have been reported in lung tissues of humans with idiopathic pulmonary hypertension (Lauer et al., 2014) and in the synovial fluid of a mouse model of rheumatoid arthritis (Lauer et al., 2013b). HA oligosaccharide treatment has been used in a mouse model of rheumatoid arthritis to irreversibly remove HC from HC-HA, thereby reducing joint inflammation (Lauer et al., 2013b). However, HC-HA complexes have not been evaluated in any developmental model of hypomyelination or in animal models of demyelination, and this is the first report to assess the effect of HA oligosaccharide treatment on cerebral inflammation, myelination, and neurological outcomes in a developmental model of brain injury. Hence, elimination of HCs from HC-HA complexes in the survivors of IVH could be a novel strategy to enhance myelination of the WM and restore neurological function.

HA oligosaccharides seem to inhibit inflammation and promote myelination by a number of mechanisms. HA oligosaccharides can interfere with HA-CD44 interactions by inducing CD44 receptor cleavage or by competitive binding to CD44 (Sugahara et al., 2003; Teriete et al., 2004). However, CD44 expression was unchanged after HA10 treatment in our model. HA oligosaccharide (HA12) impairs activated lymphocyte rolling on 

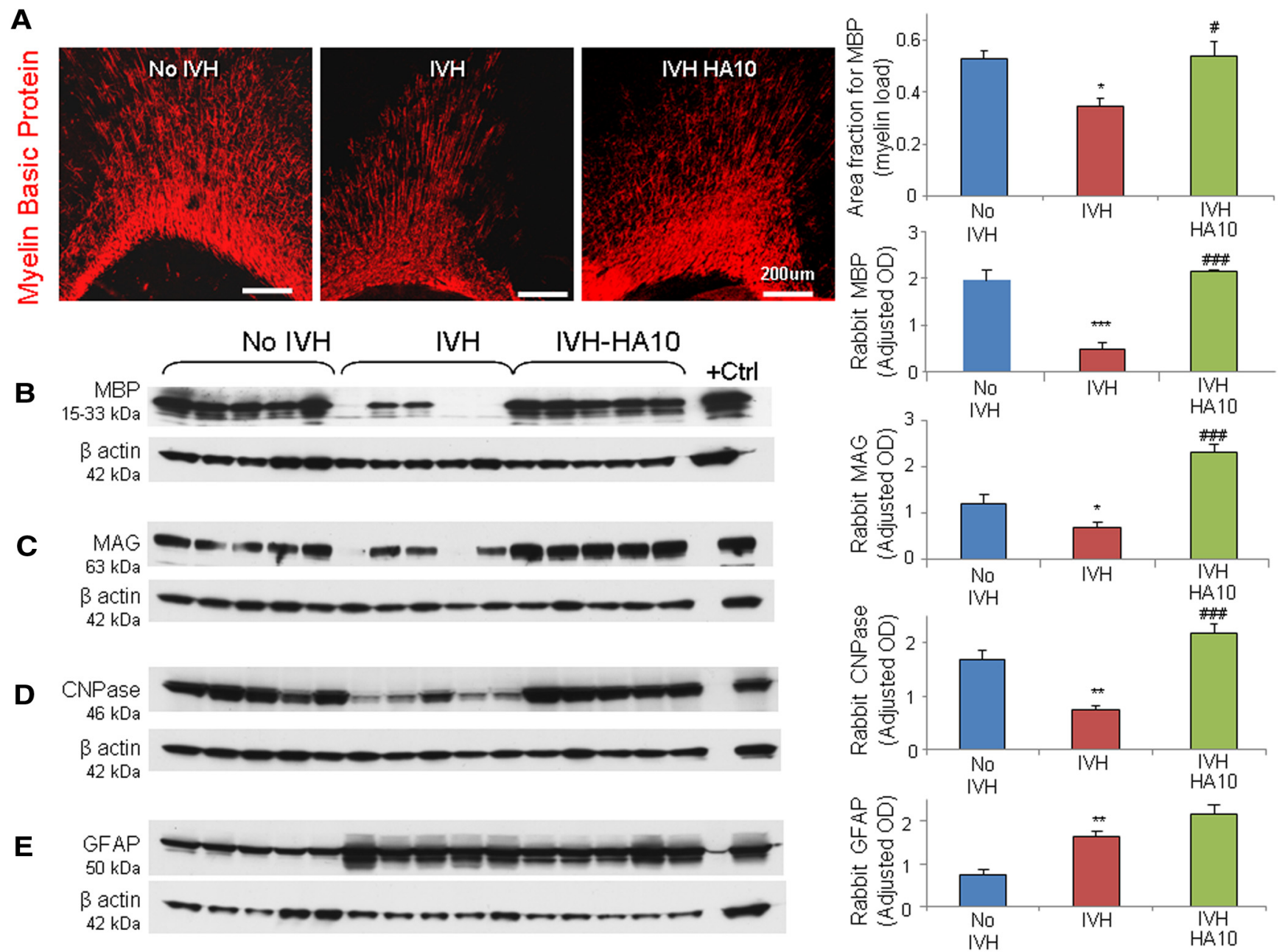

Figure 12. HA10 treatment restores myelination. $A$, Typical immunofluorescence of MBP is shown in the corona radiata of day 14 pups. The volume fraction of MBP was elevated in the corpus callosum and corona radiata of HA10-treated pups compared with vehicle controls. Scale bars: $200 \mu \mathrm{m}$. V, Ventricle. B, Representative Western blot analysis for MBP on forebrain homogenates of premature rabbit pups at P14. Adult rat brain was used as a positive control. Each lane represents a lysate from a whole coronal slice taken at the level of the midseptal nucleus. MBP expression was higher in HA10-treated pups compared with vehicle controls. C, Western blot analysis for MAG in the forebrain of pups at P14. Adult rat brain was used as a positive control. MAG expression was higher in HA10-treated pups compared with vehicle controls. D, Western blot analysis for CNPase in the forebrain of pups at P14, as indicated. Adult rat brain was used as a positive control. CNPase expression was higher in HA oligosaccharide-treated pups compared with vehicle controls. $E$, Typical Western blot analyses for GFAP on the forebrain lysate of rabbit forebrain at P14 are shown. GFAP expression was comparable between HA10-treated pups and vehicle controls. Bar graphs show the mean \pm SEM $\left(n=5\right.$ each). ${ }^{*} p<0.05,{ }^{* *} p<0.01,{ }^{* * *} p<0.001$ (pups with vs without IVH); ${ }_{p} p<0.05,{ }^{\# \# \#} p<0.001$ (vehicle- vs HA10-treated pups with IVH).

CNS endothelial cells, which is independent of CD44 and TLR4 receptors (Winkler et al., 2013). Moreover, HA12 limits demyelination in a mouse model of experimental autoimmune encephalitis (Winkler et al., 2013). Hence, HA10 oligosaccharide treatment might be alleviating IVH-induced inflammation by removing $\mathrm{HC}$ from pathological $\mathrm{HA}$, competitive binding of CD44, and displacing HA from the CD44 receptor.

HA accrues in brain lesions of premature infants with WM injury, adult stroke, multiple sclerosis, traumatic spinal cord injury, and vanishing WM disease (Back et al., 2005; Struve et al., 2005; Al'Qteishat et al., 2006; Sloane et al., 2010; Buser et al., 2012; Cargill et al., 2012). Increased levels of HA have been noted in CSF of adult patients with vascular dementia, meningoencephalitis, spinal stenosis, head injury, cerebral infarction, hydrocephalus, and encephalitis (Laurent et al., 1996; Nägga et al., 2014). Surprisingly, we did not find an increase in HA levels in infants with IVH, despite large IVH inducing extensive $\mathrm{GFAP}^{+}$gliosis in the periventricular region. Importantly, hyaluronidase treatment in rabbits with IVH degraded HA into $<100 \mathrm{kDa}$ fragments and alleviated inflammation as well as promoted myelination. Con- sistent with our study, neither recombinant human PH20 nor its generated LMW HA fragment stimulated an acute inflammatory response, but inhibited lipopolysaccharide-induced neutrophil recruitment in the air pouch model of inflammation (Huang et al., 2014). In contrast, LMW HA has induced inflammation via TLR2/4 signaling in a mouse model of lung injury (Noble et al., 1996). We attribute the differences in the findings between the studies to differences in the specific size of HA fragment used and the organ system under study, experimental context, and in vitro versus in vivo type experiments. It is possible that small HA fragments produced by hyaluronidase treatment might be alleviating inflammation and promoting myelination, similar to HA10 treatment.

Hyaluronidase treatment in infants with IVH seems promising. However, this might result in behavioral changes and adverse effects. HA modulates AMPA receptor mobility, paired-pulse depression, L-type voltage-dependent $\mathrm{Ca}^{2+}$ channel activity, and long-term potentiation (Kochlamazashvili et al., 2010). It reduces contextual fear conditioning and might induce epileptiform activity (Vedunova et al., 2013). Since HMW HA inhibits 
Table 4. Neurobehavioral evaluation of $\mathrm{HA}$ oligosaccharide- and vehicle-treated pups with IVH and controls without IVH at postnatal day 14

\begin{tabular}{|c|c|c|c|c|}
\hline System & Test & $\begin{array}{l}\text { No IVH } \\
(n=12)\end{array}$ & $\begin{array}{l}\text { IVH vehicle } \\
(n=12)\end{array}$ & $\begin{array}{l}\text { IVH, HA10 } \\
(n=10)\end{array}$ \\
\hline \multirow[t]{2}{*}{ Cranial nerve } & Aversive response to alcohol & $3(3,3)$ & $3(3,3)$ & $3(3,3)$ \\
\hline & Sucking and swallowing & $3(3,3)$ & $3(3,3)$ & $3(3,3)$ \\
\hline \multirow[t]{12}{*}{ Motor } & Motor activity & & & \\
\hline & Head & $3(3,3)$ & $3(3,3)$ & $3(3,3)$ \\
\hline & Fore legs & $3(3,3)$ & $3(3,3)$ & $3(3,3)$ \\
\hline & Hind legs & $3(3,3)$ & $3(2.25,3)^{\#}$ & $3(3,3)^{*}$ \\
\hline & Righting reflex ${ }^{a}$ & $5(5,5)$ & $4(3,5)^{\#}$ & $5(4.5,5)$ \\
\hline & Locomotion on $30^{\circ}$ inclination ${ }^{b}$ & $3(3,3)$ & $3(2,3)$ & $3(3,3)$ \\
\hline & Tone': Forelimb & $0(0,0)$ & $0(0,0)$ & $0(0,0)$ \\
\hline & Tone': Hindlimb & $0(0,0)$ & $0(0,0)$ & $0(0,0)$ \\
\hline & $\begin{array}{l}\text { Inability to hold their position } \\
\text { at } 60^{\circ} \text { inclination for } 15 \mathrm{~s} \text { or } \\
\text { less (latency to slip down } \\
\text { the slope, if }<15 \mathrm{~s} \text { ) }\end{array}$ & $0 \%$ & $33 \%$ & $10 \%$ \\
\hline & $\begin{array}{l}\text { Inability to walk more than } 60 \\
\text { inches in } 1 \mathrm{~min}(\%)\end{array}$ & $0 \%$ & $33 \%$ & $10 \%$ \\
\hline & Gait $^{d}$ & $4(4,4)$ & $3(3,4)^{\#}$ & $4(4,4)^{*}$ \\
\hline & Motor impairment ${ }^{e}$ & $0 \%$ & $25 \%$ & $10 \%$ \\
\hline \multirow[t]{2}{*}{ Sensory } & Facial touch & $3(3,3)$ & $3(3,3)$ & $3(3,3)$ \\
\hline & Pain & $3(3,3)$ & $3(3,3)$ & $3(3,3)$ \\
\hline
\end{tabular}

Values are the median and interquartile range.

${ }^{a}$ The score (range, 1-5) is the number of times the animal turned prone within $2 \mathrm{~s}$ when placed in a supine position out of five tries.

${ }^{b}$ The scoring (range, 0 -3) is as follows: 0 , does not walk; 1 , takes a few steps (less than 8 inches); 2 , walks for $9-18$ inches; 3 , walks very well beyond 18 inches.

'The scoring (range, 1-3) is as follows: 0 , no increase in tone; 1 , slight increase in tone; 2 , considerable increase in tone; 3 , limb rigid in flexion or extension.

${ }^{d}$ Gait was graded as 0 (no locomotion), 1 (crawls with trunk touching the ground for few steps and then rolls over), 2 (walks taking alternate steps, trunk low and cannot walk on inclined surface), 3 (walks taking alternate steps, cannot propel its body using synchronously the hind legs, but walks on $30^{\circ}$ inclined surface), 4 (walks, runs, and jumps without restriction and propels the body using synchronously the back legs, but limitation in speed, balance, and coordination, manifesting as clumsiness in gait), or 5 (normal walking).

${ }^{e}$ Motor impairment was defined as weakness in either fore or hind legs and a distance walked of less than 60 inches in $60 \mathrm{~s}$.

${ }^{*} p<0.05$ (vehicle-treated vs HA10-treated pups with IVH).

${ }^{\#} p<0.05$ (no IVH vs vehicle-treated pups with IVH)

and LMW HA (3-10 disaccharides) stimulates angiogenesis, hyaluronidase treatment might enhance angiogenesis and could increase the risk of IVH (Slevin et al., 2007). Importantly, intrathecal and ICV hyaluronidase have been used in children and adults with tuberculous meningitis and hydrocephalus in developing countries, demonstrating clinical efficacy and safety of intrathecal hyaluronidase treatment (Gourie-Devi and Satish, 1984; Bhagwati and George, 1986; Gourie-Devi and Satishchandra, 1991). However, the safety and efficacy of ICV hyaluronidase needs confirmation in infants and children by performing a randomized controlled clinical trial.

In conclusion, the development of IVH in preterm human infants and rabbit pups did not affect total HA concentration in the forebrain tissues, but activated HA receptors CD44 and TLR2 as well as TLR4 receptors, thereby inducing microglia infiltration and elevation in proinflammatory cytokines. There was an associated increase in TSG-6 activity and the accumulation of HC-HA complexes. Two therapeutic interventions-hyaluronidase treatment to degrade $\mathrm{HA}$ and the removal of $\mathrm{HCs}$ from HC-HA complexes by HA oligosaccharides-reduced inflammation and enhanced OPC maturation, myelination, and neurological recovery in preterm rabbits with IVH. These treatments involved ICV injection, which could be traumatic, risky, and cumbersome for use in human preterm infants. However, considering the high incidence of neurodevelopmental sequelae in infants with IVH and more invasive clinical trials performed on these infants (Whitelaw et al., 2010), intraventricular hyaluronidase might be considered for clinical trial in infants with moderate to severe IVH. Certainly, there is a need for more information on the adverse effects and pharmacokinetics of these agents. If treatment with hyaluronidase or HA oligosaccharides demonstrates efficacy and safety in preventing WM injury in premature infants, it could improve the neurologic outcome of premature infants with IVH.

\section{References}

Al'Qteishat A, Gaffney J, Krupinski J, Rubio F, West D, Kumar S, Kumar P, Mitsios N, Slevin M (2006) Changes in hyaluronan production and metabolism following ischaemic stroke in man. Brain 129:2158-2176. CrossRef Medline

Back SA, Tuohy TM, Chen H, Wallingford N, Craig A, Struve J, Luo NL, Banine F, Liu Y, Chang A, Trapp BD, Bebo BF Jr, Rao MS, Sherman LS (2005) Hyaluronan accumulates in demyelinated lesions and inhibits oligodendrocyte progenitor maturation. Nat Med 11:966-972. Medline

Ballabh P, Xu H, Hu F, Braun A, Smith K, Rivera A, Lou N, Ungvari Z, Goldman SA, Csiszar A, Nedergaard M (2007) Angiogenic inhibition reduces germinal matrix hemorrhage. Nat Med 13:477-485. CrossRef Medline

Bhagwati SN, George K (1986) Use of intrathecal hyaluronidase in the management of tuberculous meningitis with hydrocephalus. Childs Nerv Sys 2:20-25. CrossRef

Buser JR, Maire J, Riddle A, Gong X, Nguyen T, Nelson K, Luo NL, Ren J, Struve J, Sherman LS, Miller SP, Chau V, Hendson G, Ballabh P, Grafe MR, Back SA (2012) Arrested preoligodendrocyte maturation contributes to myelination failure in premature infants. Ann Neurol 71:93-109. CrossRef Medline

Bystron I, Blakemore C, Rakic P (2008) Development of the human cerebral cortex: Boulder Committee revisited. Nat Rev Neurosci 9:110-122. CrossRef Medline

Cargill R, Kohama SG, Struve J, Su W, Banine F, Witkowski E, Back SA, Sherman LS (2012) Astrocytes in aged nonhuman primate brain gray matter synthesize excess hyaluronan. Neurobiol Aging 33:830.e813-24. Medline

Chua CO, Chahboune H, Braun A, Dummula K, Chua CE, Yu J, Ungvari Z, Sherbany AA, Hyder F, Ballabh P (2009) Consequences of intraventricular hemorrhage in a rabbit pup model. Stroke 40:3369-3377. CrossRef Medline

Colón E, Shytuhina A, Cowman MK, Band PA, Sanggaard KW, Enghild JJ, Wisniewski HG (2009) Transfer of inter-alpha-inhibitor heavy chains to hyaluronan by surface-linked hyaluronan-TSG-6 complexes. J Biol Chem 284:2320-2331. CrossRef Medline

Dummula K, Vinukonda G, Chu P, Xing Y, Hu F, Mailk S, Csiszar A, Chua C, Mouton P, Kayton RJ, Brumberg JC, Bansal R, Ballabh P (2011) Bone morphogenetic protein inhibition promotes neurological recovery after intraventricular hemorrhage. J Neurosci 31:12068-12082. CrossRef Medline

Fischer M, Ehlers M (2008) Toll-like receptors in autoimmunity. Ann N Y Acad Sci 1143:21-34. CrossRef

Georgiadis P, Xu H, Chua C, Hu F, Collins L, Huynh C, Lagamma EF, Ballabh P (2008) Characterization of acute brain injuries and neurobehavioral profiles in a rabbit model of germinal matrix hemorrhage. Stroke 39: 3378-3388. CrossRef Medline

Gourie-Devi M, Satish P (1984) Intrathecal hyaluronidase treatment of chronic spinal arachnoiditis of noninfective etiology. Surg Neurol 22: 231-234. CrossRef Medline

Gourie-Devi M, Satishchandra P (1991) Hyaluronidase as an adjuvant in the management of tuberculous spinal arachnoiditis. J Neurol Sci 102: 105-111. CrossRef Medline

Guan H, Nagarkatti PS, Nagarkatti M (2011) CD44 Reciprocally regulates the differentiation of encephalitogenic Th1/Th17 and Th2/regulatory T cells through epigenetic modulation involving DNA methylation of cytokine gene promoters, thereby controlling the development of experimental autoimmune encephalomyelitis. J Immunol 186:6955-6964. CrossRef Medline

Haserodt S, Aytekin M, Dweik RA (2011) A comparison of the sensitivity, specificity, and molecular weight accuracy of three different commercially 
available Hyaluronan ELISA-like assays. Glycobiology 21:175-183. CrossRef Medline

Huang Z, Zhao C, Chen Y, Cowell JA, Wei G, Kultti A, Huang L, Thompson CB, Rosengren S, Frost GI, Shepard HM (2014) Recombinant human hyaluronidase $\mathrm{PH} 20$ does not stimulate an acute inflammatory response and inhibits lipopolysaccharide-induced neutrophil recruitment in the air pouch model of inflammation. J Immunol 192:5285-5295. CrossRef Medline

Jiang D, Liang J, Noble PW (2011) Hyaluronan as an immune regulator in human diseases. Physiol Rev 91:221-264. CrossRef Medline

Kessaris N, Fogarty M, Iannarelli P, Grist M, Wegner M, Richardson WD (2006) Competing waves of oligodendrocytes in the forebrain and postnatal elimination of an embryonic lineage. Nat Neurosci 9:173-179. CrossRef Medline

Kim JA, Ko JH, Ko AY, Lee HJ, Kim MK, Wee WR, Lee RH, Fulcher SF, Oh JY (2014) TSG-6 protects corneal endothelium from transcorneal cryoinjury in rabbits. Invest Ophthalmol Vis Sci 55:4905-4912. CrossRef Medline

Kochlamazashvili G, Henneberger C, Bukalo O, Dvoretskova E, Senkov O, Lievens PM, Westenbroek R, Engel AK, Catterall WA, Rusakov DA, Schachner M, Dityatev A (2010) The extracellular matrix molecule hyaluronic acid regulates hippocampal synaptic plasticity by modulating postsynaptic L-type $\mathrm{Ca}(2+)$ channels. Neuron 67:116-128. CrossRef Medline

Konat GW, Kielian T, Marriott I (2006) The role of Toll-like receptors in CNS response to microbial challenge. J Neurochem 99:1-12. Medline

Lauer ME, Mukhopadhyay D, Fulop C, de la Motte CA, Majors AK, Hascall VC (2009) Primary murine airway smooth muscle cells exposed to poly $(\mathrm{I}, \mathrm{C})$ or tunicamycin synthesize a leukocyte-adhesive hyaluronan matrix. J Biol Chem 284:5299-5312. CrossRef Medline

Lauer ME, Cheng G, Swaidani S, Aronica MA, Weigel PH, Hascall VC (2013a) Tumor necrosis factor-stimulated gene-6 (TSG-6) amplifies hyaluronan synthesis by airway smooth muscle cells. J Biol Chem 288: 423-431. CrossRef Medline

Lauer ME, Glant TT, Mikecz K, DeAngelis PL, Haller FM, Husni ME, Hascall VC, Calabro A (2013b) Irreversible heavy chain transfer to hyaluronan oligosaccharides by tumor necrosis factor-stimulated gene-6. J Biol Chem 288:205-214. CrossRef Medline

Lauer ME, Aytekin M, Comhair SA, Loftis J, Tian L, Farver CF, Hascall VC, Dweik RA (2014) Modification of hyaluronan by heavy chains of interalpha-inhibitor in idiopathic pulmonary arterial hypertension. J Biol Chem 289:6791-6798. CrossRef Medline

Laurent UB, Laurent TC, Hellsing LK, Persson L, Hartman M, Lilja K (1996) Hyaluronan in human cerebrospinal fluid. Acta Neurol Scand 94: 194-206. Medline

Lehnardt S, Lehmann S, Kaul D, Tschimmel K, Hoffmann O, Cho S, Krueger C, Nitsch R, Meisel A, Weber JR (2007) Toll-like receptor 2 mediates CNS injury in focal cerebral ischemia. J Neuroimmunol 190:28-33. CrossRef Medline

Marret S, Delpech B, Delpech A, Asou H, Girard N, Courel MN, Chauzy C, Maingonnat C, Fessard C (1994) Expression and effects of hyaluronan and of the hyaluronan-binding protein hyaluronectin in newborn rat brain glial cell cultures. J Neurochem 62:1285-1295. Medline

Milner CM, Day AJ (2003) TSG-6: a multifunctional protein associated with inflammation. J Cell Sci 116:1863-1873. CrossRef Medline

Nägga K, Hansson O, van Westen D, Minthon L, Wennstrom M (2014) Increased Levels of Hyaluronic Acid in Cerebrospinal Fluid in Patients with Vascular Dementia. J Alzheimers Dis 42:1435-1441. Medline

Nicolay DJ, Doucette JR, Nazarali AJ (2007) Transcriptional control of oligodendrogenesis. Glia 55:1287-1299. CrossRef Medline

Noble PW, McKee CM, Cowman M, Shin HS (1996) Hyaluronan fragments activate an NF-kappa B/I-kappa B alpha autoregulatory loop in murine macrophages. J Exp Med 183:2373-2378. CrossRef Medline

Petrey AC, de la Motte CA (2014) Hyaluronan, a crucial regulator of inflammation. Front Immunol 5:101. Medline

Preston M, Gong X, Su W, Matsumoto SG, Banine F, Winkler C, Foster S, Xing R, Struve J, Dean J, Baggenstoss B, Weigel PH, Montine TJ, Back SA,
Sherman LS (2013) Digestion products of the PH20 hyaluronidase inhibit remyelination. Ann Neurology 73:266-280. CrossRef

Rivest S (2009) Regulation of innate immune responses in the brain. Nature Rev Immunol 9:429-439. CrossRef

Slevin M, Krupinski J, Gaffney J, Matou S, West D, Delisser H, Savani RC, Kumar S (2007) Hyaluronan-mediated angiogenesis in vascular disease: uncovering RHAMM and CD44 receptor signaling pathways. Matrix Biol 26:58-68. CrossRef Medline

Sloane JA, Batt C, Ma Y, Harris ZM, Trapp B, Vartanian T (2010) Hyaluronan blocks oligodendrocyte progenitor maturation and remyelination through TLR2. Proc Natl Acad Sci U S A 107:11555-11560. CrossRef Medline

Stern R, Asari AA, Sugahara KN (2006) Hyaluronan fragments: an information-rich system. Eur J Cell Biol 85:699-715. CrossRef Medline

Struve J, Maher PC, Li YQ, Kinney S, Fehlings MG, Kuntz C 4th, Sherman LS (2005) Disruption of the hyaluronan-based extracellular matrix in spinal cord promotes astrocyte proliferation. Glia 52:16-24. CrossRef Medline

Sugahara KN, Murai T, Nishinakamura H, Kawashima H, Saya H, Miyasaka M (2003) Hyaluronan oligosaccharides induce CD44 cleavage and promote cell migration in CD44-expressing tumor cells. J Biol Chem 278: 32259-32265. CrossRef Medline

Tang SC, Arumugam TV, Xu X, Cheng A, Mughal MR, Jo DG, Lathia JD, Siler DA, Chigurupati S, Ouyang X, Magnus T, Camandola S, Mattson MP (2007) Pivotal role for neuronal Toll-like receptors in ischemic brain injury and functional deficits. Proc Natl Acad Sci U S A 104:1379813803. CrossRef Medline

Teriete P, Banerji S, Noble M, Blundell CD, Wright AJ, Pickford AR, Lowe E, Mahoney DJ, Tammi MI, Kahmann JD, Campbell ID, Day AJ, Jackson DG (2004) Structure of the regulatory hyaluronan binding domain in the inflammatory leukocyte homing receptor CD44. Mol cell 13:483-496. CrossRef Medline

Tuohy TM, Wallingford N, Liu Y, Chan FH, Rizvi T, Xing R, Bebo B, Rao MS, Sherman LS (2004) CD44 overexpression by oligodendrocytes: a novel mouse model of inflammation-independent demyelination and dysmyelination. Glia 47:335-345. CrossRef Medline

Vedunova M, Sakharnova T, Mitroshina E, Perminova M, Pimashkin A, Zakharov Y, Dityatev A, Mukhina I (2013) Seizure-like activity in hyaluronidase-treated dissociated hippocampal cultures. Front Cell Neurosci 7:149. Medline

Vinukonda G, Csiszar A, Hu F, Dummula K, Pandey NK, Zia MT, Ferreri NR, Ungvari Z, LaGamma EF, Ballabh P (2010) Neuroprotection in a rabbit model of intraventricular haemorrhage by cyclooxygenase-2, prostanoid receptor-1 or tumour necrosis factor-alpha inhibition. Brain 133:22642280. CrossRef Medline

Watanabe J, Shetty AK, Hattiangady B, Kim DK, Foraker JE, Nishida H, Prockop DJ (2013) Administration of TSG-6 improves memory after traumatic brain injury in mice. Neurobiol Dis 59:86-99. CrossRef Medline

Whitelaw A, Jary S, Kmita G, Wroblewska J, Musialik-Swietlinska E, Mandera M, Hunt L, Carter M, Pople I (2010) Randomized trial of drainage, irrigation and fibrinolytic therapy for premature infants with posthemorrhagic ventricular dilatation: developmental outcome at 2 years. Pediatrics 125:e852-858. CrossRef Medline

Winkler CW, Foster SC, Itakura A, Matsumoto SG, Asari A, McCarty OJ, Sherman LS (2013) Hyaluronan oligosaccharides perturb lymphocyte slow rolling on brain vascular endothelial cells: implications for inflammatory demyelinating disease. Matrix Biol 32:160-168. CrossRef Medline

Zhang R, Liu Y, Yan K, Chen L, Chen XR, Li P, Chen FF, Jiang XD (2013) Anti-inflammatory and immunomodulatory mechanisms of mesenchymal stem cell transplantation in experimental traumatic brain injury. J Neuroinflammation 10:106. CrossRef Medline

Zhuo L, Kanamori A, Kannagi R, Itano N, Wu J, Hamaguchi M, Ishiguro N, Kimata K (2006) SHAP potentiates the CD44-mediated leukocyte adhesion to the hyaluronan substratum. J Biol Chem 281:20303-20314. CrossRef Medline 